ARTICLE

https://doi.org/10.1038/s41467-019-13697-y

\title{
Structural insights into ubiquitin recognition and Ufd1 interaction of Npl4
}

\author{
Yusuke Sato $1,2,3,5,7$, Hikaru Tsuchiya ${ }^{4,7}$, Atsushi Yamagata1,2,3,6, Kei Okatsu'1,2, Keiji Tanaka4, \\ Yasushi Saeki ${ }^{4 \star} \&$ Shuya Fukai (1) ${ }^{1,2,3 \star}$
}

Npl4 is likely to be the most upstream factor recognizing Lys48-linked polyubiquitylated substrates in the proteasomal degradation pathway in yeast. Along with Ufd1, Npl4 forms a heterodimer (UN), and functions as a cofactor for the Cdc48 ATPase. Here, we report the crystal structures of yeast Npl4 in complex with Lys48-linked diubiquitin and with the Npl4binding motif of Ufd1. The distal and proximal ubiquitin moieties of Lys48-linked diubiquitin primarily interact with the $\mathrm{C}$-terminal helix and $\mathrm{N}$-terminal loop of the Npl4 C-terminal domain (CTD), respectively. Mutational analysis suggests that the CTD contributes to linkage selectivity and initial binding of ubiquitin chains. Ufd1 occupies a hydrophobic groove of the Mpr1/Pad1 N-terminal (MPN) domain of Npl4, which corresponds to the catalytic groove of the MPN domain of JAB1/MPN/Mov34 metalloenzyme (JAMM)-family deubiquitylating enzyme. This study provides important structural insights into the polyubiquitin chain recognition by the Cdc48-UN complex and its assembly.

\footnotetext{
${ }^{1}$ Institute for Quantitative Biosciences, The University of Tokyo, Tokyo 113-0032, Japan. ${ }^{2}$ Synchrotron Radiation Research Organization, The University of Tokyo, Tokyo 113-0032, Japan. ${ }^{3}$ Department of Computational Biology and Medical Sciences, Graduate School of Frontier Sciences, The University of Tokyo, Chiba 277-8562, Japan. ${ }^{4}$ Laboratory of Protein Metabolism, Tokyo Metropolitan Institute of Medical Science, Tokyo 156-8506, Japan. ${ }^{5}$ Present address: Center for Research on Green Sustainable Chemistry, Tottori University, Tottori 680-8582, Japan. ${ }^{6}$ Present address: RIKEN Center for Biosystems Dynamics Research, Kanagawa 230-0045, Japan. ${ }^{7}$ These authors contributed equally: Yusuke Sato, Hikaru Tsuchiya. *email: saeki-ys@igakuken.or.jp; fukai@iam.utokyo.ac.jp
} 
biquitin ( $\mathrm{Ub}$ ) is an essential posttranslational modifier conserved from yeast to mammals ${ }^{1-3}$. Typically, the Cterminal glycine residue of $\mathrm{Ub}$ is covalently attached to lysine residues of substrate proteins. Ub itself is also a substrate for ubiquitylation, and forms a covalently linked Ub chain. The Ub chain linked via Lys48 (K48 chain) is the primary targeting signal for proteasomal degradation ${ }^{1-5}$. Polyubiquitylated proteins in isolation can be direct targets of the proteasome, whereas those embedded into membranes or assembled into multisubunit complexes need to be extracted or segregated by the conserved AAA-family ATPase Cdc48 (yeast) or p97/VCP (metazoan) with the aid of its cofactor complex, Ufd1-Npl4 (UN), prior to the proteasomal degradation ${ }^{6-11}$. The cellular function or localization of the Cdc48/p97 ATPase is controlled by many cofactors. The $\mathrm{UN}$ heterodimer is one of the best-characterized cofactors ${ }^{12-15}$.

The Cdc48/p97 ATPase consists of an N-terminal (N) domain and two ATPase domains (D1 and D2). D1 and D2 form a hexameric double ring with a central pore ${ }^{16}$. The conformation of the $\mathrm{N}$ domain relative to the D1-D2 ring is coupled to the nucleotide state of $\mathrm{D} 1$; the $\mathrm{N}$ domain is located above the $\mathrm{D} 1$ ring in the ATP state (up conformation), and is coplanar with the D1 ring in the ADP state (down conformation) ${ }^{17-21}$. In addition, relative rotations between the D1 and D2 rings occur upon binding of ATP to D2. Although understanding the mechanism of the substrate translocation accompanied with the Ub chain binding and ATP hydrolysis has been a long-standing problem, recent structural and functional studies provided important mechanistic insights into reacting steps of the Cdc48/p97-UN complex $^{22}$. The UN heterodimer captures the substrate-attached $\mathrm{Ub}$ chain at the initial step ${ }^{15}$.

$\mathrm{Npl} 4$ is responsible for recognition of the substrate-attached $\mathrm{Ub}$ chain, and thus likely mediates a Ub chain-associated reacting step $^{15}$. The mammalian Npl4 (also known as NPLOC4) consists of a Ubx-like (UBXL) domain, zinc-finger domain (previously designated as zf-Npl4), Mpr1/Pad1 N-terminal (MPN) domain, C-terminal domain (CTD), and NZF domain. The mammalian $\mathrm{Npl} 4$ binds to Ub chains without linkage specificity through the NZF domain. On the other hand, yeast Npl4 lacks the NZF domain, and binds specifically to K48 chains via zf-Npl4-MPN$\mathrm{CTD}^{15,23,24}$. The MPN domain of Npl4 topologically resembles the catalytic domain of the JAB1/MPN/Mov34 metalloenzyme (JAMM)-family deubiquitylating enzyme (DUB) ${ }^{19}$, which accommodates the C-terminal tail of Ub. Therefore, the groove corresponding to the catalytic site of JAMM DUBs ${ }^{25,26}$ has been proposed as a potential Ub-binding site of Npl4. However, a recent cryo-EM analysis of the substrate-engaged Cdc48-UN complex at 3.9 $\AA$ resolution has shown that the K48 chain on the substrate does not interact with the groove of yNpl4 MPN but with CTD and other regions of MPN ${ }^{22}$. Nevertheless, due to the low local resolution, the density for Ufd 1 was not interpreted with the atomic model and the interaction between Npl4 and the two folded Ub moieties has not been characterized in detail ${ }^{22}$.

Here, we present the crystal structures of yeast Npl4 (zf-Npl4MPN-CTD) in complex with Lys48-linked diubiquitin $\left(\mathrm{K} 48-\mathrm{Ub}_{2}\right)$ and with Ufd 1 at 2.55 and $1.58 \AA$ resolutions, respectively. The crystal structures reveal that the distal and proximal $\mathrm{Ub}$ (Ub ${ }^{\text {dist }}$ and Ubprox, respectively) moieties of $\mathrm{K} 48-\mathrm{Ub}_{2}$ interact with the C-terminal helix and N-terminal loop (N loop) of Npl4 CTD, respectively, and that Ufd1 binds to the groove of Npl4 corresponding to the catalytic groove of JAMM. In vitro and in vivo mutational analyses confirm that the Npl4 MPN groove is required for binding to Ufd1 but not to K48 chains. Also, Npl4 mutants that are designed to disrupt the Ub binding show that the interaction between the Npl4 C-terminal helix and $\mathrm{Ub}$ is critical for the Ub chain binding and stimulation of the ATPase activity of the Cdc48-UN complex. Moreover, we reveal that mutations on the proximal Ub-binding site of Npl4 affect its linkage-specificity. Our crystallographic and biochemical studies provide essentially important insights to understand the reaction mechanism of the Cdc48/p97-UN complex.

\section{Results}

Structure of $\mathrm{Npl} 4$ in complex with $\mathrm{K} 48-\mathrm{Ub}_{2}$. The region containing residues $113-580$ of Saccharomyces cerevisiae Npl4 (yNpl4113-580, with the prefix " $y$ " indicating Saccharomyces cerevisiae proteins) specifically recognizes $\mathrm{K} 48$ chains in vitro (Fig. 1) ${ }^{15}$. The triple E123A K124A E125A mutation was introduced to reduce excess surface conformational entropy ${ }^{27}$. The yNpl4113-580 (E123A K124A E125A) protein yielded high-quality crystals, and its structure was determined at $1.72 \AA$ resolution by the single-wavelength anomalous diffraction (SAD) method using the zinc edge (Table 1). We also attempted to crystallize yNpl4 $4^{113-580}$ in complex with $\mathrm{K} 48$ chains. Although $\mathrm{K} 48-\mathrm{Ub}_{2}$, $\mathrm{K} 48-\mathrm{Ub}_{3}, \mathrm{~K} 48-\mathrm{Ub}_{4}$, and $\mathrm{K} 48-\mathrm{Ub}_{5}$ were tested for crystallization of the complex, only $\mathrm{K} 48-\mathrm{Ub}_{2}$ was successfully co-crystallized with yNpl $4^{113-580}$. Eventually, we determined the crystal structure of yNpl4 ${ }^{113-580}$ in complex with selenomethionine (SeMet)-labeled $\mathrm{K} 48-\mathrm{Ub}_{2}$ at $2.55 \AA$ resolution (Fig. $2 \mathrm{a}$ and Table 1 ). The structure was determined by the molecular replacement method using yNpl4 ${ }^{113-580}$ alone as the search model. Although molecular replacement using Ub (PDB 1UBQ [https://doi.org/10.2210/ pdblubq/pdb] $)^{28}$ as the search model was unsuccessful, we found residual electron density corresponding to $\mathrm{K} 48-\mathrm{Ub}_{2}$ and manually built the model of $\mathrm{K} 48-\mathrm{Ub}_{2}$. The final model contains one yNpl4113-580-K48-Ub $4_{2}$ complex and one isolated yNpl4 ${ }^{113-580}$ molecule in the asymmetric unit. We here note that the electron density of $\mathrm{K} 48-\mathrm{Ub}_{2}$ is weak, especially of Ubprox (Supplementary Fig. 1a). The electron density of the yNpl4interacting part of Ubprox is observed, whereas the solvent exposed part of Ubprox is obscured (Supplementary Fig. 1a, b). To confirm the positions of $\mathrm{Ub}^{\text {dist }}$ and Ubprox, we replaced Pro19 Val26 or Ile30 of Ub with SeMet and calculated the anomalous difference Fourier map in the $\mathrm{yNpl} 4^{113-580}-\mathrm{K} 48-\mathrm{Ub}_{2}$ complex (Supplementary Fig. 1c). Although some signals derived from SeMet were indistinguishable or not detected, we detected the signals derived from SeMet1, SeMet19, and SeMet26 of the Ub dist and SeMet1, SeMet26, and SeMet30 of the Ubprox.

The yNpl4 ${ }^{113-580}$ structure consists of three subdomains, $\mathrm{zf}-$ Npl4, MPN, and CTD, similarly to the previously reported structures of $\mathrm{Npl}^{19,22}$. The yNpl4 MPN subdomain consists of the MPN core and two insertions, Ins-1 (residues 287-317) and Ins-2 (residues 403-462), similarly to other MPN DUBs. The $\mathrm{Ub}^{\text {dist }}$ moiety contacts the CTD subdomain of yNpl4 with a buried surface area of $460 \AA^{2}$ (Fig. 2b). The Ubprox moiety contacts the CTD and MPN subdomains of yNpl4 with a buried surface area of $468 \AA^{2}$. Although the electron density corresponding to the C-terminal five residues (Arg-Leu-Arg-Gly-Gly) of Ub $^{\text {dist }}$ and the Lys48 side chain of Ubprox are invisible (Fig. 2c), the $\mathrm{Ca}-\mathrm{Ca}$ distance between Leu71 of $\mathrm{Ub}^{\text {dist }}$ and Lys48 of Ubprox $(11 \AA)$ is within the range of those in the previously reported crystal structures of K48 chains in complex with their specific effectors (SARS PLpro ${ }^{29}$, AIRAPL ${ }^{30}$, and Mindy-1 ${ }^{31}$; 9-20 A). Furthermore, we examined the $\mathrm{yNpl} 4-\mathrm{K} 48-\mathrm{Ub}_{2}$ crystals by SDSPAGE (Supplementary Fig. 1d), and confirmed that the linkage of $\mathrm{K} 48-\mathrm{Ub}_{2}$ was retained in the crystals. Therefore, we concluded that the isopeptide bond connects $\mathrm{Ub}^{\text {dist }}$ and Ubprox in the present $\mathrm{yNpl} 4-\mathrm{K} 48-\mathrm{Ub}_{2}$ structure. No large conformational difference was observed between yNpl4113-580 alone and yNpl4 ${ }^{113-580}-\mathrm{K} 48-\mathrm{Ub}_{2}$, except for the region comprising residues 290-300 in Ins-1, whose conformation is constrained by crystal packing (Supplementary Fig. 2a). 


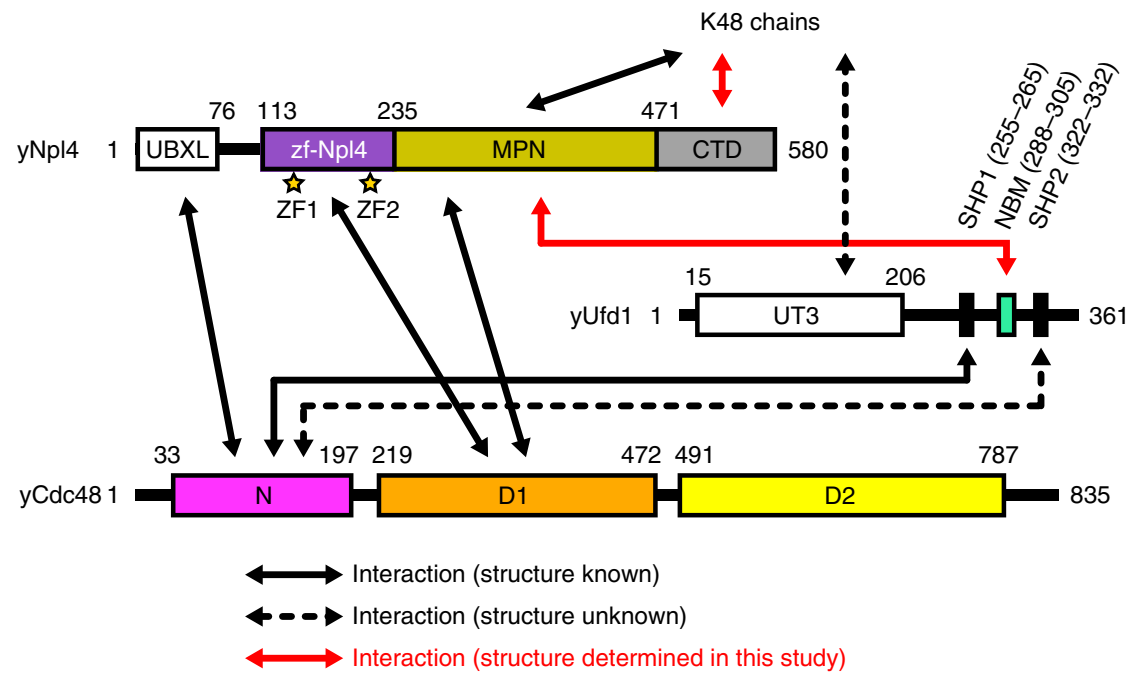

Fig. 1 Domain compositions of S. cerevisiae Npl4, Ufd1, and Cdc48. The zf-Npl4, MPN, and CTD subdomains of yNpl4 are purple, dark yellow, and gray, respectively. ZF1 and ZF2 of yNpl4 are indicated by yellow stars. The NBM region of Ufd1 is turquoise. The N, D1, and D2 domains of Cdc48 are magenta, orange, and yellow, respectively.

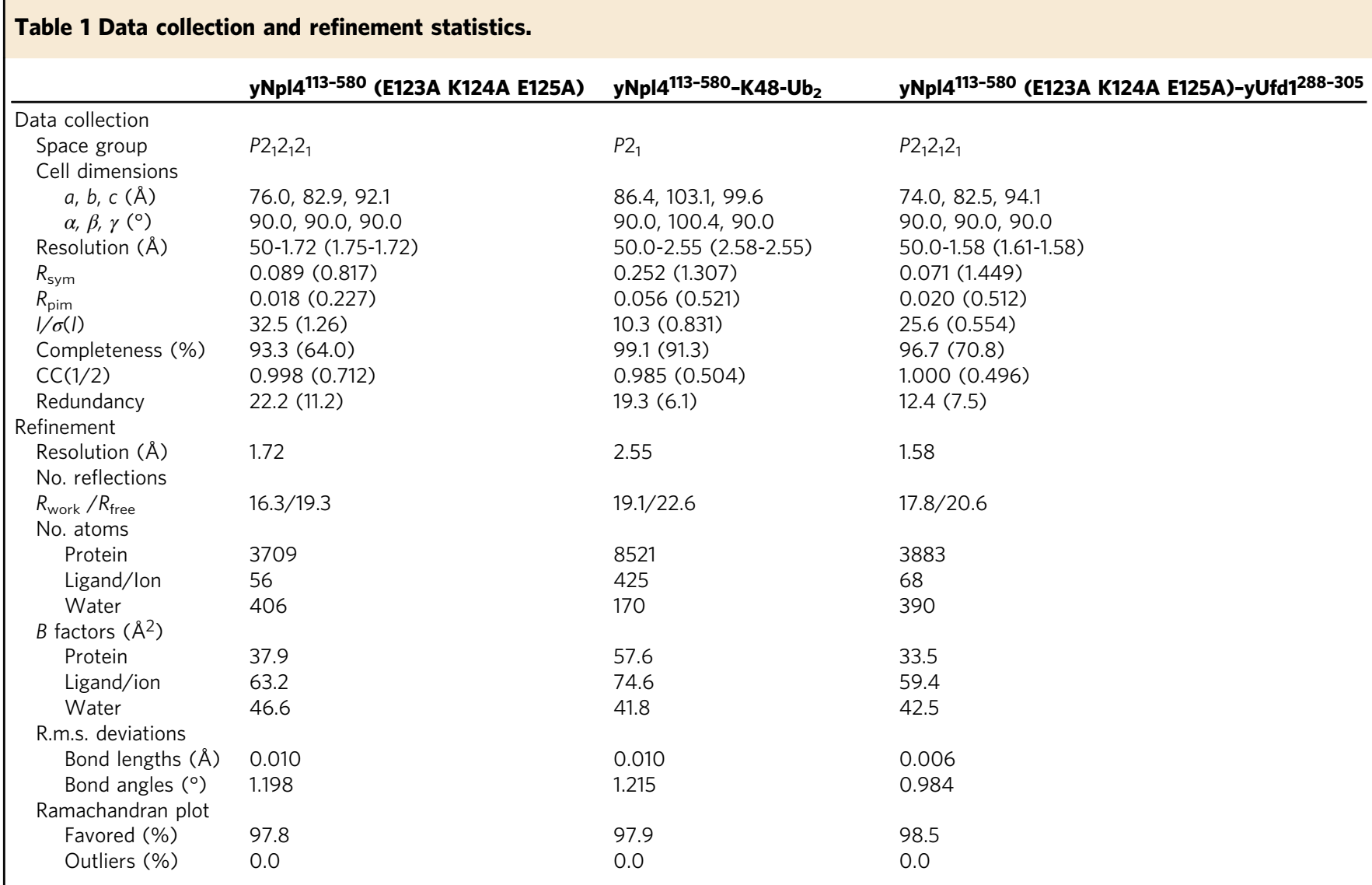

Values in parentheses are for highest-resolution shell

The C-terminal helix of Npl4 plays a key role in Ub binding. $\mathrm{Ub}^{\text {dist }}$ of $\mathrm{K} 48-\mathrm{Ub}_{2}$ interacts primarily with the C-terminal helix of yNpl4 CTD (Fig. 2b). Met574 and Ile575 form a hydrophobic surface to interact with the Ile44-centered hydrophobic patch of $\mathrm{Ub}^{\text {dist }}$. This hydrophobic interaction is further stabilized by hydrogen bonds between Thr571 of yNpl4 and the main-chain $\mathrm{NH}$ groups of Ala46 and Gly47 in Ubdist. Adjacent to the C- terminal helix-mediated interactions, Tyr501 and Ile538 of yNpl4 project into a hydrophobic pocket formed by Leu8, His68, and Val70 of $\mathrm{Ub}^{\text {dist }}$. To assess the roles of these interactions in the $\mathrm{Ub}$ chain recognition by $\mathrm{Npl} 4$, we examined the binding of the T571A, M574A, M574Q, and I575A mutants of yNpl4 to K48 chains by surface-plasmon resonance (SPR) spectroscopy (Table 2). The SPR analysis of mutant yNpl4 was performed 
a

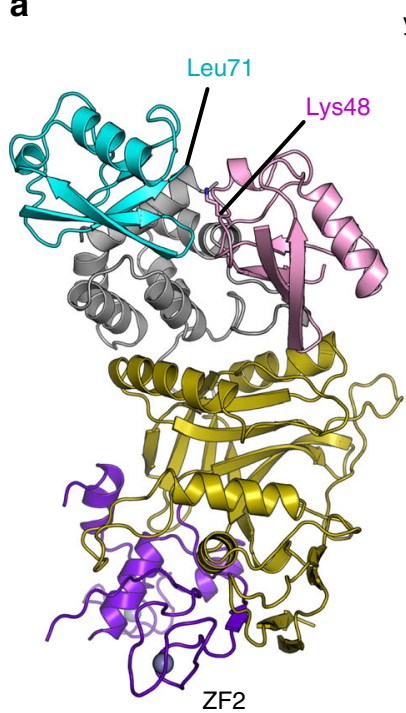

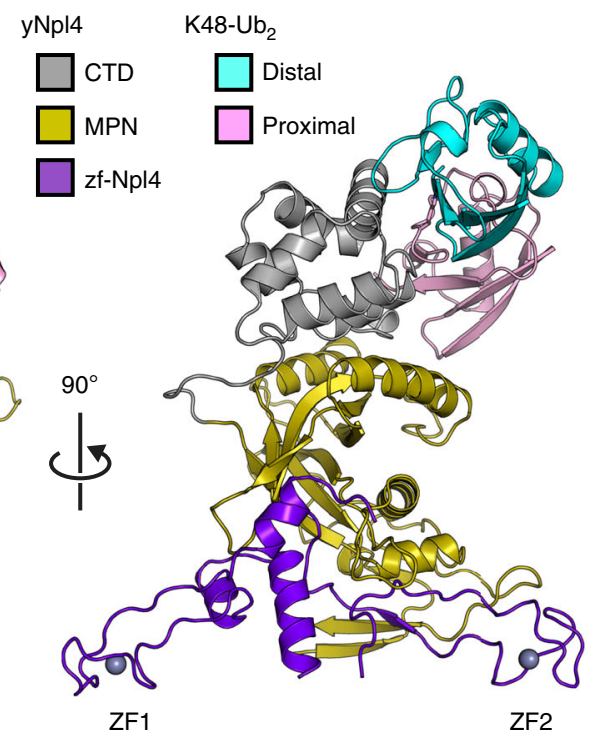

d

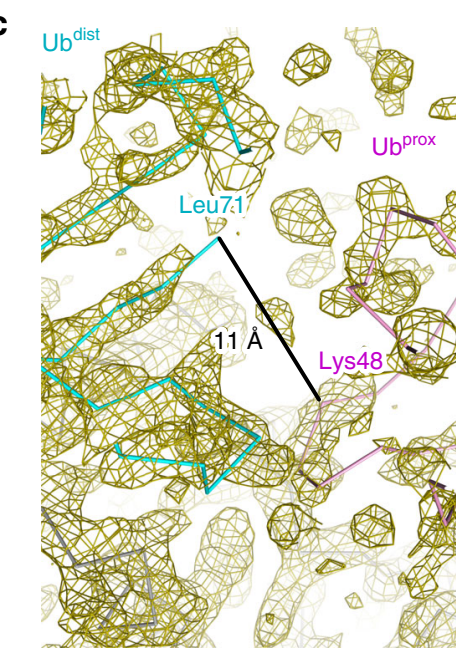

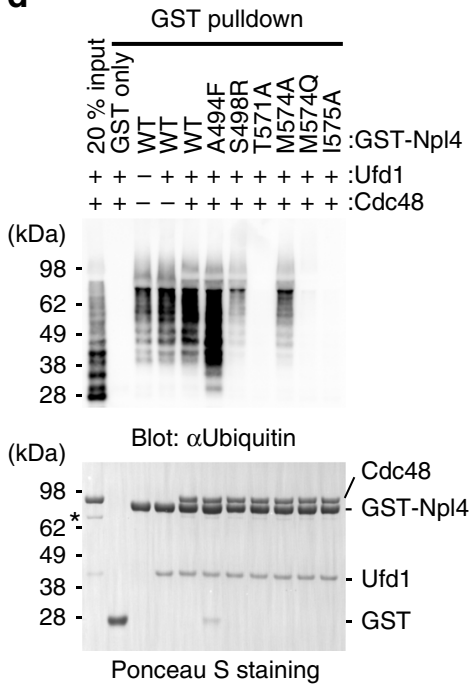

b
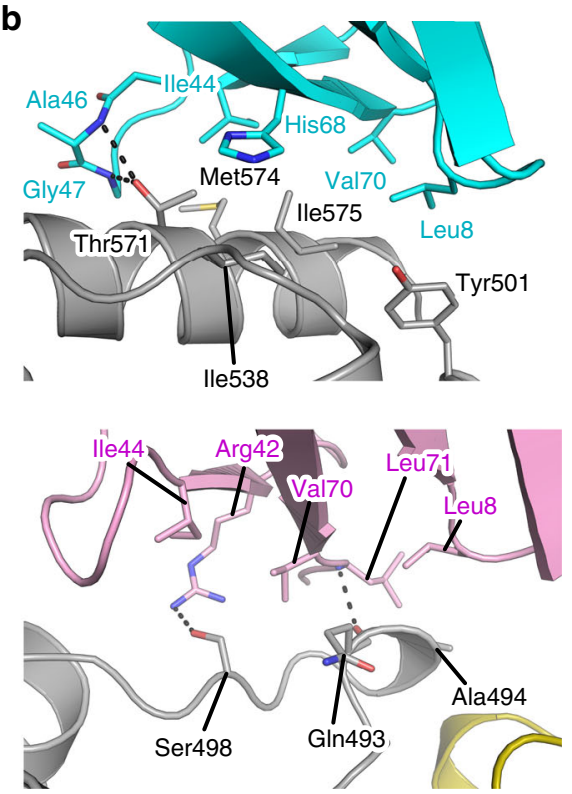

e

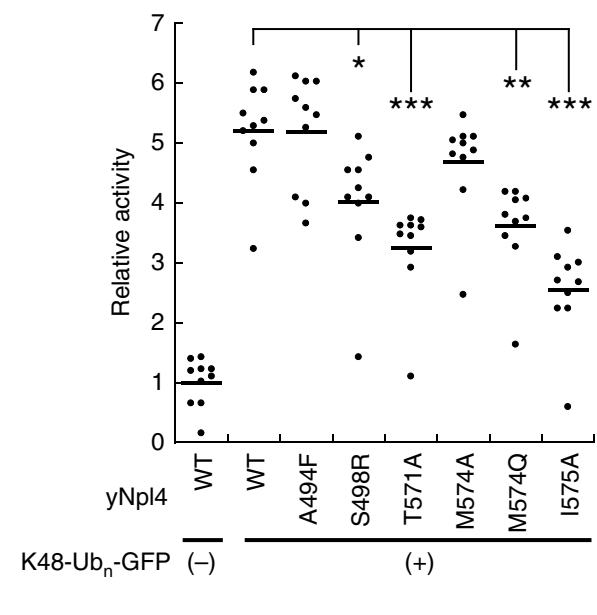

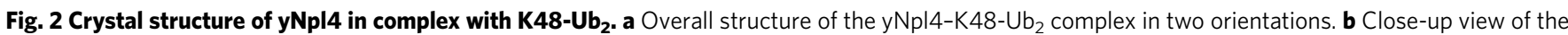
interactions between y Npl4 and $\mathrm{K} 48-\mathrm{Ub}_{2}$. Hydrogen bonds are shown as black dotted lines. c Close-up view of the area around the isopeptide linkage of $\mathrm{K} 48-\mathrm{Ub}_{2}$. A $2 \mathrm{~F}_{\mathrm{o}}-\mathrm{F}_{\mathrm{c}}$ composite omit map is shown as an olive mesh contoured at $1 \sigma$ level. $\mathbf{d}$ Analysis of the binding between $\mathrm{K} 48 \mathrm{chains}$ and the $\mathrm{Cdc} 48-\mathrm{UN}$ complex containing wild-type or mutant GST-yNpl4 by pulldown assays. The bound K48 chains were detected by immunoblotting with anti-Ub antibody (upper panel). Blot membranes were stained with Ponceau S (lower panel). In all, 20\% input means 20\% of the volume of the sample (K48 chain, Cdc48, and yUfd1) that was mixed with the GST-yNpl4-bound glutathione resin. Asterisks indicate contamination. This experiment was repeated with distinct samples (Supplementary Fig. 3a). Source data are provided as a Source Data file. e ATP hydrolysis rates of the Cdc48-UN complex containing wild-type or mutant $\mathrm{yNpl} 4$ with or without $\mathrm{K} 48-\mathrm{Ub}_{\mathrm{n}}$-GFP. The rates were normalized to the average of the ATP hydrolysis rates of the wild-type Cdc48-UN complex without K48-Ubn - GFP. The line represents the mean of the rates after normalization (mean values; $n=10$ independent experiments; ${ }^{\star} P<0.05 ;{ }^{\star \star} P<0.01 ;$ ${ }^{\star \star \star} P<0.001$ from Tukey's test).

using $\mathrm{K} 48-\mathrm{Ub}_{4}$ because the affinity for $\mathrm{K} 48-\mathrm{Ub}_{2}$ was too low to be analyzed. Longer $\mathrm{Ub}$ chains bind to their effectors with higher affinity in general ${ }^{30,32,33}$. The T571A mutation of yNpl4 $113-580$ decreased the affinity for $\mathrm{K} 48-\mathrm{Ub}_{4}$ to $5.0 \%$ of the wild-type affinity (Table 2). The I575A mutation of yNpl4 ${ }^{113-580}$ decreased the affinity to an unmeasurable level. The M574A mutation of yNpl4 $4^{113-580}$ had a weak effect and decreased the affinity for K48$\mathrm{Ub}_{4}$ to $43 \%$ of the wild-type affinity. On the other hand, The M574Q mutation had a greater effect than the M574A mutation, and decreased the affinity for $\mathrm{K} 48-\mathrm{Ub}_{4}$ to $9.1 \%$ of the wild-type affinity. This is likely because the hydrophilic side chain of Gln inhibits the hydrophobic interaction between $y \mathrm{~Np} 14^{113-580}$ and $\mathrm{Ub}^{\text {dist }}$. We further analyzed the affinities between yNpl4 ${ }^{113-580}$ mutants and K63- or M1-Ub . The T571A, M574A, M574Q, or I575A mutation decreased the affinity for K63- or $\mathrm{M} 1-\mathrm{Ub}_{4}$ to an unmeasurable level (Table 2). These results suggest that the yNpl4-Ub dist interface primarily contributes to the affinity for Ub chains rather than the linkage specificity.

Next, the binding between the Cdc48-UN complex and K48 chains was analyzed by GST pulldown assays. GST-yNpl4 mutants were immobilized to glutathione beads and incubated with Cdc48, yUfd1, and K48 chains. After washing, the bound 
Table 2 Binding affinity of Npl4 for K48, K63, or M1 chains.

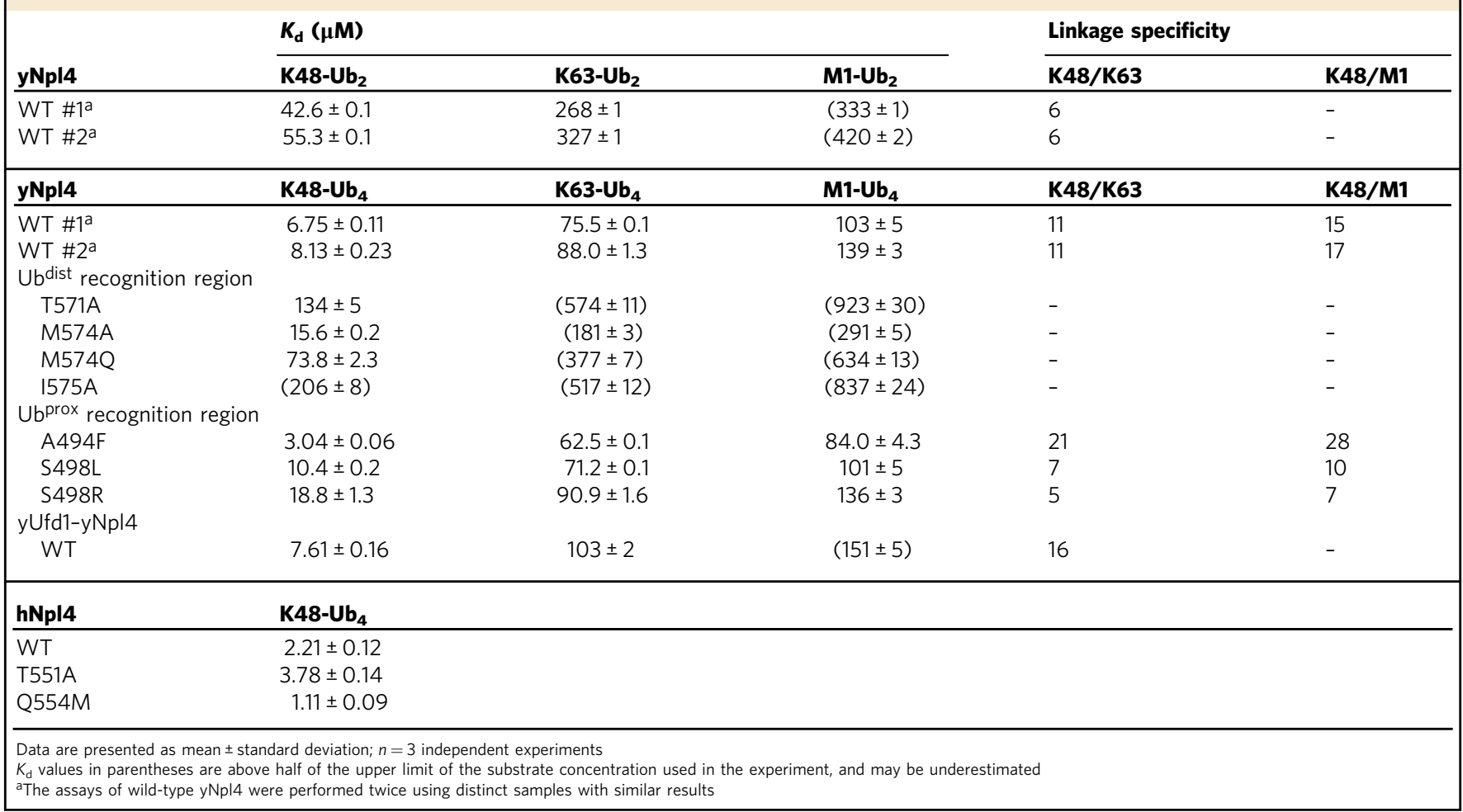

proteins were eluted with LDS-loading buffer and analyzed by immunoblotting with anti-Ub antibody (Fig. 2d and Supplementary Fig. 3a). In this assay, yUfd1 or Cdc48 had a little effect on the binding to K48 chains, and the UN or Cdc48-UN complex formation was not affected by the mutations of yNpl4 examined in this study. On the other hands, the T571A, M574Q, or I575A mutation, which had a severe effect on the $\mathrm{K} 48-\mathrm{Ub}_{4}$ binding, completely abolished the binding of Cdc48-UN for K48 chains. The M574A mutation, which had a mild effect on the affinity for K48-Ub $\mathrm{Ub}_{4}$, did not completely eliminate the binding. Similar GST pulldown assays using GST-yUfd1 instead of GST-yNpl4 showed consistent results (Supplementary Fig. 3b). To further assess the functional significance of the yNpl4-Ubdist interaction in vivo, mutant yNpl4-3xFLAG was expressed under its own promoter in $n$ pl4A cells. yNpl4-3xFLAG was immunoprecipitated with antiFLAG antibody, and co-immunoprecipitation of Lys48-linked polyubiquitylated proteins were analyzed with anti-K48 chain antibody (Supplementary Fig. 3c). In agreement with the result of the in vitro binding assay, the T571A, M574Q, or I575A mutation decreased the amount of co-immunoprecipitated Lys48-linked polyUb conjugates, although these mutations had little effect on Cdc48 binding.

Previous studies showed that Ufd1 binds to K48 chains through its UT3 domain ${ }^{24,34}$. However, our pulldown analysis failed to detect the binding of yUfd1 to K48 chains (Supplementary Fig. 3b). We next analyzed the binding between the GST-yNpl4 and K48 chains on increasing concentrations of yUfd1 by GST pulldown assay but did not observe substantial effects (Supplementary Fig. 3d). SPR analysis using the purified yUfd1-yNpl4113-580 complex and $\mathrm{K} 48-\mathrm{Ub}_{4}$ also showed that the presence of $\mathrm{yUfd} 1$ has little effect on the affinity of $\mathrm{yNpl} 4$ for $\mathrm{K}_{48}-\mathrm{Ub}_{4}$ (Table 2). Although the $K_{\mathrm{d}}$ value of yUfd1 for polyUb has not been estimated, that for monoUb was estimated to be within the range of 1-2 $\mathrm{mM}^{34}$. This value is much higher than the $K_{\mathrm{d}}$ value of $\mathrm{yNpl} 4$ for $\mathrm{K} 48-\mathrm{Ub}_{2}$ or $\mathrm{K} 48-\mathrm{Ub}_{4}$ (Table 2 ), suggesting that the affinity of $\mathrm{yUfd} 1$ for $\mathrm{K} 48$ chains is much lower than that for yNpl4. Collectively, these in vitro and in vivo results suggest that the C-terminal helix of yNpl4 is the key determinant for $\mathrm{Ub}$ chain recognition by the Cdc48-UN complex.

The N loop of Npl4 CTD contributes to K48 chain specificity. $\mathrm{Ub}^{\text {prox }}$ of $\mathrm{K} 48-\mathrm{Ub}_{2}$ mainly interacts with the $\mathrm{N}$ loop of $\mathrm{yNpl} 4$ CTD (residues 493-501) (Fig. 2b). The $\mathrm{N}$ loop of yNpl4 CTD interacts with the hydrophobic patch formed by Leu8, Ile44, and Val70 of Ubprox. Ser498 of yNpl4 forms a hydrogen bond with Arg42 of Ubprox. The main-chain CO group of Gln493 in yNpl4 forms a hydrogen bond with the main-chain $\mathrm{NH}$ group of Leu71 in Ubprox. The functional importance of these interactions was confirmed by SPR analyses using the A494F, S498L, and S498R mutants of $\mathrm{His}_{6}-\mathrm{yNpl} 4^{113-580}$ (Table 2). The S498L mutation of yNpl4 had a little effect on the affinity for K48-, K63-, and M1$\mathrm{Ub}_{4}$, whereas the S498R mutation of $\mathrm{yNpl} 4$ decreased the affinity for $\mathrm{K} 48-\mathrm{Ub}_{4}$ to $36 \%$ of the wild-type affinity (Table 2). These results suggest that the $\mathrm{N}$ loop of $\mathrm{yNpl} 4 \mathrm{CTD}$ is a binding site for Ubprox, although the contribution of the $\mathrm{N}$ loop to $\mathrm{K} 48-\mathrm{Ub}_{4}$ binding is smaller than that of the C-terminal helix. Reflecting this, gaps are found in the interface between yNpl4 and Ubprox. One of the gaps is located between Ala494 of yNpl4 and Leu8 of Ubprox. The Phe replacement of Ala494 in yNpl4 increased the affinity of $\mathrm{yNpl} 4$ for $\mathrm{K} 48-\mathrm{Ub}_{4} 2.2$-fold (Table 2). It is likely that the bulky side chain of Phe filled the gap and increased the affinity. The involvement of the $\mathrm{N}$ loop of yNpl4 CTD in the Ubprox recognition was further supported by GST pulldown assays using the Cdc48-UN complex containing a GST-yNpl4 mutant: the $\mathrm{A} 494 \mathrm{~F}$ mutation of $\mathrm{yNpl} 4$ enhanced the binding between Cdc48-UN and K48 chains, whereas the S498R mutation decreased it (Fig. 2d). Similar results were obtained even when the GST tag was fused to Ufd1 instead of Npl4 (Supplementary Fig. 3b).

The S498R or A494F mutation hardly affected the affinity for $\mathrm{K} 63-$ or $\mathrm{M} 1-\mathrm{Ub}_{4}$, in contrast to that for $\mathrm{K} 48-\mathrm{Ub}_{4}$, and thereby 
changed the linkage specificity (represented by the reciprocal ratio of dissociation constants; Table 2). The affinity of wild-type $\mathrm{yNpl} 4$ for $\mathrm{K} 48-\mathrm{Ub}_{4}$ was 11 and 15 times higher than that for K63or $\mathrm{M} 1-\mathrm{Ub}_{4}$, respectively. The S498R mutation of the $\mathrm{yNpl} 4$ decreased the linkage specificity. In contrast, the A494F mutant of yNpl4 increased the linkage specificity because this mutant increases the affinity for $\mathrm{K} 48-\mathrm{Ub}_{4}$ but hardly affects that for K63or $\mathrm{M} 1-\mathrm{Ub}_{4}$ (Table 2). These results indicate that the interaction between $\mathrm{Ub}^{\text {prox }}$ and the $\mathrm{N}$ loop of $\mathrm{yNpl} 4 \mathrm{CTD}$ contributes to the specificity of yNpl4 to K48 chains.

K48 chain-CTD binding stimulates the Cdc48 ATPase activity. Lys48-linked polyubiquitylated GFP (K48-Ub $\left.\mathrm{Ub}_{\mathrm{n}}-\mathrm{GFP}\right)$ has been used as a model substrate that can stimulate the ATP hydrolysis of the Cdc48-UN complex ${ }^{13,14}$. To evaluate the coupling between the binding to a K48 chain and ATP hydrolysis in the Cdc48-UN complex, we analyzed the rate of the ATP hydrolysis by the Cdc48-UN complex containing mutant yNpl4. Addition of K48$\mathrm{Ub}_{\mathrm{n}}$-GFP increased the ATP hydrolysis rate of the Cdc48-UN complex, depending on the affinity of $\mathrm{yNpl} 4$ for K48 chains (Fig. 2e and Supplementary Fig. 3e). For instance, K48-Ub $\mathrm{U}_{\mathrm{n}} \mathrm{GFP}$ increased the ATP hydrolysis rate of the Cdc48-UN complex approximately 5-fold. The T571A, M574Q, or I575A mutation at the yNpl4-Ub dist interface, which severely decreases the affinity for K48 chains, decreased the ATP hydrolysis rate. The S498R mutation at the yNpl4-Ubprox interface, which shows a mild effect on the affinity, also decreased the ATPase hydrolysis rate but less than the T571A, M574Q, or I575A mutation. These results indicate that the stimulation of the ATPase activity of the Cdc48-UN complex requires the binding activity of $\mathrm{yNpl} 4 \mathrm{CTD}$ to K48 chains.

Structure of Npl4 in complex with Ufd1. Npl4 and Ufd1 can form a heterodimer, even in the absence of $\mathrm{Cdc} 48 / \mathrm{p} 97$ or a polyubiquitylated substrate. The mechanism of the Ufd1-Npl4 interaction remains unclear, although residues $258-275$ of human Ufd1 (hUfd1258-275, with the prefix " $h$ " indicating the human protein; equivalent to residues 288-305 of yUfd (yUfd1 $\left.{ }^{288-305}\right)$ ) have been assigned as the Npl4-binding motif (NBM) (Fig. 1) $)^{35}$. Indeed, fluorescence anisotropy-based affinity measurement using FlAsH-labeled yUfd1288-305 showed that $\mathrm{yUfd}^{288-305}$ binds to yNpl4 with $K_{\mathrm{d}}$ of $85.7 \mathrm{nM}$ (Table 3 ). To further reveal the structural basis of the interaction between Npl4 and Ufd1, we determined the crystal structure of yNpl4113-580 (E123A K124A E125A) in complex with yUfd1 $1^{288-305}$ (Fig. 3a, b and Table 1). The structure was determined by the molecular replacement method using yNpl4 ${ }^{113-580}$ alone as the search model. In the crystal, yNpl4 ${ }^{113-580}$ formed a stoichiometric complex with yUfd $1^{288-305}$. No large conformational difference was observed between $y N^{113-580}$ alone and the $\mathrm{yUfd}^{288-305}$-bound yNpl4113-580 (Supplementary Fig. 2b).

In the complex, residues $298-300$ of yUfd1 NBM form a $\beta$ sheet with Ins-1 of yNpl4 (Fig. 3c, d). Leu296, Phe326, Leu353, Met357, Phe419, Pro420, and Tyr424 of yNpl4 form a hydrophobic groove to accommodate Pro289, Leu292, Leu294, Gly297, Leu299, Phe301, Phe303, and Met305 of yUfd1. Mutations of all these residues except for Pro420 of yNpl4 and Pro289 of yUfd1 were examined by fluorescent anisotropy-based binding analysis (Table 3). The mutation of Leu296, Leu353, or Tyr424 of yNpl4 or Leu292, Gly297, Leu299, or Phe301 of yUfd1 severely affected the affinity between yUfd1288-305 and yNpl4113-580. The Ala replacement of Leu292, Leu299, or Phe301 in yUfd $1{ }^{288-305}$ decreased the affinity to $1.6,0.68$, or $0.41 \%$ of the wild-type affinity, respectively. The Leu, Arg, or Tyr replacement of Gly297 of yUfd1 ${ }^{288-305}$ decreased the affinity for $y \mathrm{Npl}^{113-580}$ to $14,0.41$, or $4.0 \%$ of the wild-type affinity, respectively. The

\begin{tabular}{|c|c|c|}
\hline & $K_{\mathrm{d}}(\mathrm{nM})$ & Fold of increase \\
\hline WT \#19 & $85.7 \pm 2.6$ & 1 \\
\hline WT \#2a & $99.4 \pm 6.7$ & 1 \\
\hline \multicolumn{3}{|c|}{ yNpl4 WT + yUfd1 mutants } \\
\hline L292A & $5292 \pm 788$ & 62 \\
\hline L294A & $527 \pm 98$ & 6 \\
\hline E296A & $437 \pm 48$ & 5 \\
\hline G297L & $619 \pm 90$ & 7 \\
\hline G297R & $20896 \pm 2066$ & 244 \\
\hline G297Y & $2136 \pm 362$ & 25 \\
\hline L299A & $12677 \pm 639$ & 148 \\
\hline F301A & $20770 \pm 2906$ & 242 \\
\hline F303A & $1766 \pm 116$ & 21 \\
\hline M305A & $261 \pm 8$ & 3 \\
\hline \multicolumn{3}{|c|}{ yNpl4 mutants + yUfd1 WT } \\
\hline L296A & $7019 \pm 60$ & 82 \\
\hline F326A & $257 \pm 27$ & 3 \\
\hline L353A & $1380 \pm 81$ & 16 \\
\hline M357A & $164 \pm 14$ & 2 \\
\hline R364A & $314 \pm 41$ & 4 \\
\hline F419A & $158 \pm 18$ & 2 \\
\hline Y424A & $8447 \pm 823$ & 99 \\
\hline
\end{tabular}

Data are presented as mean \pm standard deviation; $n=3$ independent experiments

aThe assays of wild-type y $\mathrm{Npl} 4$ and $\mathrm{yUfd} 1-\mathrm{FlAsH}$ were performed twice using distinct samples with similar results

backbone dihedral angles $\varphi$ and $\psi$ of Gly297 are $90.1^{\circ}$ and $-8.5^{\circ}$, respectively. MolProbity ${ }^{36}$, a standard program for protein structure validation, judges these angles as favored angles for Gly but allowed angles for non-Gly/Pro residues. Gly is more favorable than non-Gly/Pro residues at residue 297. yUfd1 NBM is kinked at Gly297, thereby fitting into the hydrophobic groove in the MPN subdomain of yNpl4 (Fig. 3b). Gly297 and Phe301 of yUfd1 are conserved from yeast to human, whereas Leu299 of yUfd1 is replaced by functionally equivalent hydrophobic residues among other eukaryotes (Fig. 3c). Although the sequence of Ufd 1 NBM is variable among eukaryotes, this $\mathrm{Gx} \Phi \mathrm{xF}$ motif ( $\mathrm{x}$ and $\Phi$ represent any amino acids and hydrophobic amino acids, respectively) is well conserved, reflecting its functional importance (Fig. 3c). The Ala replacement of Leu296, Phe326, Leu353, or Tyr424 of yNpl4 ${ }^{113-580}$ decreased the affinity to $1.2,33,6.2$, or $1.0 \%$ of the wild-type affinity, respectively (Table 3 ). Notably, Leu296, Phe326, and Tyr424 of yNpl4 are conserved in hNpl4 (Supplementary Fig. 4). Leu296 and Phe326 of yNpl4 interact with Leu299 and Phe301 of yUfd1, which occupy the third and fifth positions of the GxФxF motif, and Tyr424 of yNpl4 hydrophobically interacts with the main-chain CO group of Ala290 and the Ca atom of Lys291 in yUfd1. These hydrophobic interactions are likely conserved from yeast to human. In addition to the hydrophobic interactions, Arg364 and Thr418 of yNpl4 form hydrogen bonds with Glu296 and the main-chain $\mathrm{NH}$ group of Ala290 in yUfd1, respectively. The E296A mutation of yUfd $1^{288-305}$ or the R364A mutation of yNpl4 ${ }^{113-580}$ decreased the affinity to $20 \%$ or $27 \%$ of the wild-type affinity, respectively (Table 3). These residues are not conserved between yeast and human. The hydrophilic interactions between Npl4 and Ufd1 may be variable among species.

Ufd1 does not overlap with K48 chain on Npl4. The catalytic groove in the MPN domain of JAMM-family DUBs accommodates the C-terminal tail of Ub for cleavage $25,26,37$. The first insertion in the JAMM core (i.e., Ins-1) forms a $\beta$-sheet with the C-terminal tail of Ub in the catalytic groove (Fig. 3d). Npl4 is enzymatically inactive but has Ins-1 and a groove similar to the 
a

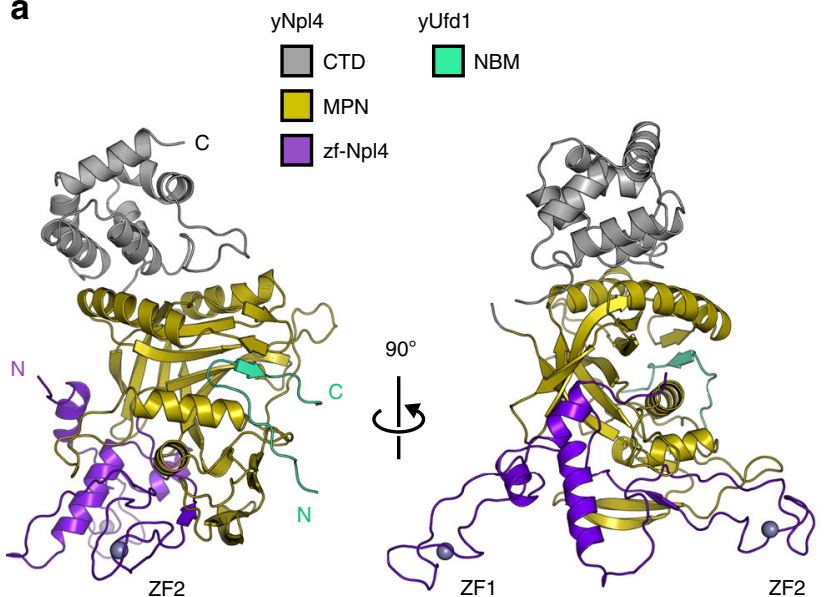

C

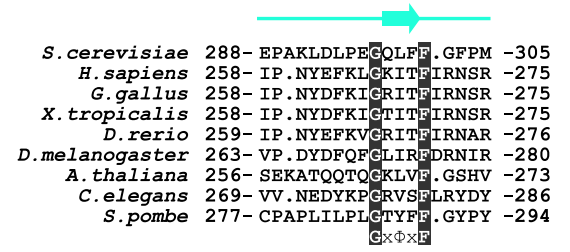

d

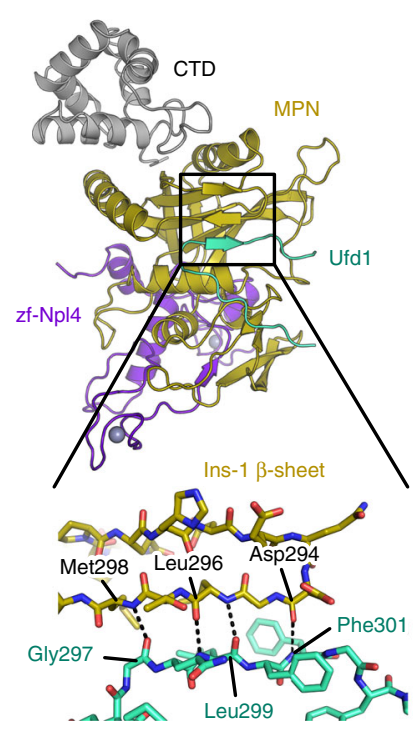

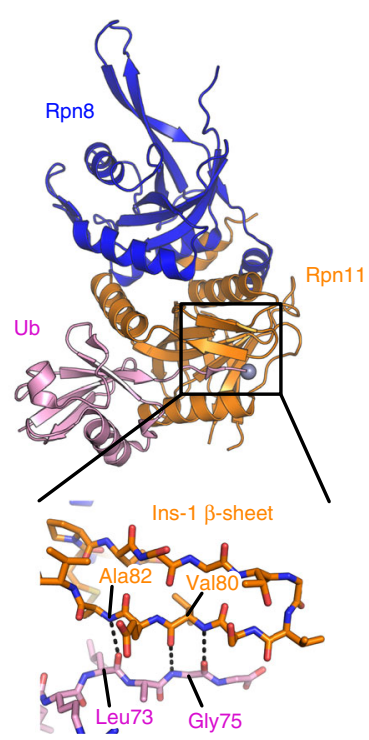

b

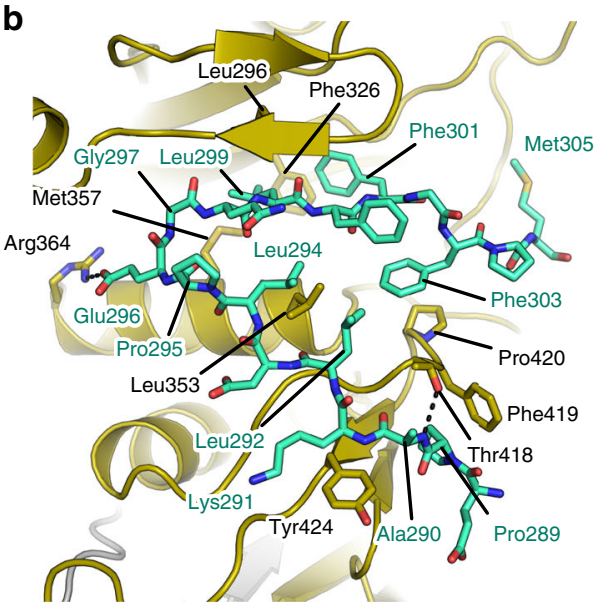

e
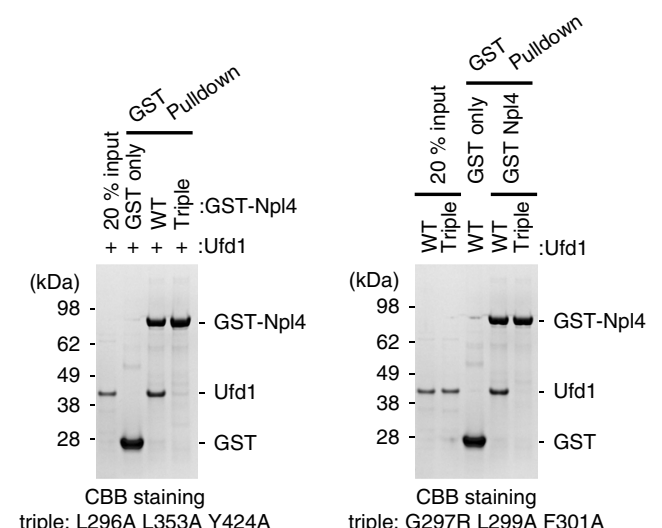

f

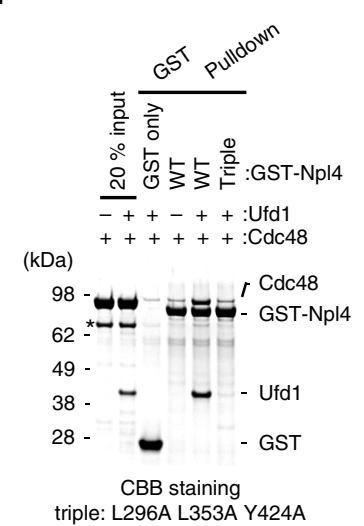

Fig. 3 Crystal structure of yNpl4 in complex with yUfd1. a Overall structure of the yNpl4-yUfd1 complex in two orientations. $\mathbf{b}$ Close-up view of the interaction between yNpl4 and yUfd1. Hydrogen bonds are shown as black dotted lines. c Sequence alignment of the NBM region of Ufd1. The position of the $\beta$-strand in the NBM region in the yNpl4-yUfd1 structure is shown above the sequences. $\mathbf{d}$ Comparison of the MPN region of the yNpl4-yUfd1 and Rpn8-Rpn11-Ub (PDB 5U4P [https://doi.org/10.2210/pdb5u4p/pdb]) 25 complexes. The coloring scheme of the yNpl4-yUfd1 complex is the same as that in Fig. 3a. Rpn8, Rpn11, and Ub of the Rpn8-Rpn11-Ub complex are blue, orange, and pink, respectively. Hydrogen bonds are shown as black dotted lines. $\mathbf{e}, \mathbf{f}$ Analysis of the binding between GST-yNpl4 and yUfd1 alone (e) or yUfd1-Cdc48 (f) by pulldown assays. The results from the triple mutant of yNpl4 (L296A L353A Y424) (left) or the triple mutant of yUfd1 (G297R L299A F301A) (right) are shown. In all, 20\% input means $20 \%$ of the volume of the sample (yUfd1 and/or Cdc48) that was mixed with the GST-yNpl4-bound glutathione resin. Asterisks indicate contamination. The bound proteins were analyzed by SDS-PAGE and stained with Coomassie brilliant blue. Source data are provided as a Source Data file.

catalytic groove of JAMM-family DUBs ${ }^{19}$. Therefore, this groove and Ins-1 have been assumed to form a potential Ub chainbinding site. However, the present yNpl4 $4^{113-580}-\mathrm{yUfd} 1^{288-305}$ structure reveals that the groove of the MPN domain accommodates yUfd1 NBM (Fig. 3d). The Npl4-bound yUfd1 does not overlap with $\mathrm{K} 48-\mathrm{Ub}_{2}$ in the $\mathrm{yNpl} 4-\mathrm{K} 48-\mathrm{Ub}_{2}$ complex. Consistently, yUfd1 did not inhibit the binding of K48 chains to yNpl4 (Fig. 2d, Supplementary Fig. 3d). In addition, the recently reported cryo-EM structure of the substrate-engaged Cdc48-UN showed that the bound K48 chain and Ufd1 do not overlap with 
each other. In spite of the similarity of the MPN domains of Npl4 and JAMM DUBs, the functions of Ins-1 and the groove are different between them.

Cdc48-UN assembly depends on the Ufd1-Npl4 interaction. To analyze the relationship between the Ufd1-Npl4 interaction and Cdc48-UN assembly, we first screened for mutations that effectively inhibit the interaction between yUfd 1 and yNpl4 by GST pulldown experiments with full-length proteins. Although single point mutations of yNpl4 or yUfd1 hardly affected the yNpl4-yUfd1 interaction (Supplementary Fig. 5a, b), triple mutations of yNpl4 (L296A L353A Y424A) or yUfd1 (G297R L299A F301A) completely inhibited the formation of the UN heterodimer, respectively (Fig. 3e and Supplementary Fig. 5a, b). The triple mutant yNpl4 was confirmed to bind to K48 chains as well as wild-type yNpl4 (Supplementary Fig. 5c). Next, we analyzed the effect of yNpl4 or yUfd1 mutations on the Cdc48-UN assembly by GST pulldown experiments and found that the triple mutants of either yNpl4 or yUfd1 substantially reduced the formation of the Cdc48-UN complex (Fig. 3f and Supplementary Fig. 5d). Thus, the UN heterodimer formation facilitates the Cdc48-UN assembly, although both yNpl4 and yUfd1 can directly bind to Cdc48.

To further assess the functional significance of the Ufd1-Npl4 interaction in vivo, mutated Npl4-3xFLAG or Ufd1-3xFLAG was expressed in $n p l 4 \Delta$ or $u f d 1 \Delta$ cells, respectively. We examined the accumulation of $\mathrm{Ub}$ conjugates in total cell lysate in these cells. The $n p l 4-1$ temperature-sensitive strain was also examined as an Npl4-deficient control. The levels of Ub conjugates were not changed in the cells that expressed yNpl4 or yUfd1 single-point mutants (Supplementary Fig. 5e). In contrast, in the cells expressing the triple mutant of yNpl4 or yUfd1, we observed a substantial accumulation of Ub conjugates, which was comparable to that in the npl4-1 cell (Supplementary Fig. 5e). Correspondingly, these triple mutant-expressing cells displayed a temperature-sensitive growth phenotype, although it was milder than the npl4-1 phenotype (Supplementary Fig. 5f). These findings indicate that the Ufd1-Npl4 interaction is important for the degradation of $\mathrm{Ub}$ conjugates and for the cell growth.

CTD of human Npl4 is involved in binding to K48 chains. Npl4 is a highly conserved protein from yeast to mammals. In mammalian Npl4, the NZF domain located in its C-terminus binds to K48 chains and Lys63-linked Ub chains (K63 chains). The human Npl4 (hNpl4) mutant lacking the NZF domain hardly binds to Ub chains ${ }^{15}$. On the other hand, the Ascomycota and Plantae Npl4 proteins lack the NZF domain in their C-termini (Supplementary Fig. 6a, b). The Npl4 proteins from $S$. cerevisiae and $C$. thermophilum bind to K48 chains via the zf-Npl4-MPN-CTD domain ${ }^{19}$. Multiple amino-acid sequence alignment including yNpl4 and hNpl4 shows that the Ub ${ }^{\text {dist }}$-interacting residues in the C-terminal helix of the CTD are partially conserved between yeast and human; Thr571 of yNpl4 is conserved, and Ile575 of yNpl4 is replaced with Leu in hNpl4, which should be functionally equivalent to Ile (Supplementary Fig. 6a). Intriguingly, the T551A mutation of hNpl4 (equivalent to Thr571 of yNpl4) decreased the affinity for $\mathrm{K} 48-\mathrm{Ub}_{4}$ to $58 \%$ of the wild-type affinity (Table 2). On the other hand, Met574 of yNpl4 is replaced with Gln in hNpl4 and with Ala in S. pombe. The M574Q mutation of yNpl4 decreased the affinity for $\mathrm{K} 48-\mathrm{Ub}_{4}$ to $9.1 \%$ of the wild-type affinity, whereas the M574A mutation decreased it to just $43 \%$ (Table 2). Reciprocally, the Q554M mutation of hNpl4 (equivalent to Met574 of yNpl4) increased the affinity for $\mathrm{K} 48-\mathrm{Ub}_{4}$ about two-fold (Table 2). These findings suggest that the C-terminal helix of hNpl4 CTD is also involved in binding to a Ub chain like that of yNpl4 CTD.

hNpl4 NZF can compensate for the defect of yNpl4 CTD. To assess the functional equivalence between yNpl4 CTD and hNpl4 NZF in the context of the Ub chain binding and the Cdc48 ATPase activity, we investigated engineered yNpl4 proteins, where the NZF domain of hNpl4 (including the linker region between the CTD and NZF domains) was fused to the C-terminal end of the full-length yNpl4 (yNpl4-NZF) with or without mutations deficient in binding to Ub chains (T571A or I575A). GST pulldown analysis showed that yNpl4-NZF bound to both K48 and K63 chains, even with the T571A or I575A mutation, indicating that the NZF fusion can rescue the defect of Ub chain binding of the CTD mutations (Fig. 4a, b and Supplementary Fig. 6c). Next, we analyzed whether the yNpl4-NZF proteins support the K48 chain-dependent ATPase activity of the Cdc48 complex (Fig. 4c and Supplementary Fig. 6d). The NZF fusion to wild-type yNpl4 did not enhance the ATPase activity above the wild-type level, probably because the affinity of wild-type yNpl4 for K48 chains is sufficient to stimulate the full ATPase activity of Cdc48. On the other hand, the NZF fusion completely recovered the decrease in the ATPase stimulation by the T571A or I575A mutation, suggesting that $\mathrm{yNpl} 4 \mathrm{CTD}$ and hNpl4 NZF are functionally equivalent. This also raises the possibility that the enhancement of ATP hydrolysis is independent of Lys48-linkage specificity. However, K63 chains did not stimulate the ATPase activity of the Cdc48-UN complex containing yNpl4-NZF, which can bind to K63 chains as well as to K48 chains (Fig. 4c). Thus, the stimulation of the ATPase activity of the Cdc48-UN complex actually depends on Lys48-linkage specificity of yNpl4. Considering that Lys48-linkage specificity of yNpl4 CTD is dispensable for the stimulation of the ATP hydrolysis, one may expect that yNpl4, yUfd1, and/or Cdc48 have additional Lys48linkage-specific recognition site(s) besides Npl4 CTD. The cryoEM structure of the substrate-engaged Cdc48-UN has shown that yNpl4 MPN binds to unfolded Ub, which bridges between Ubprox and the central pore of $\mathrm{Cdc} 48^{22}$ (Supplementary Fig. 6e). This binding may be related to the K48 chain-specific stimulation of the ATPase activity of Cdc48.

\section{Discussion}

In this study, we determined the crystal structure of the yNpl4 $4^{113-580}-\mathrm{K} 48-\mathrm{Ub}_{2}$ complex. Both Ub ${ }^{\text {dist }}$ and Ubprox interact with yNp14 CTD, which is a newly identified Ub-binding domain (Fig. 5a). The C-terminal helix of yNpl4 CTD is the primary Ubbinding site of yNpl4. Among the previously reported Ub-binding domains (UBDs), Ub-interacting motif (UIM), motif interacting with Ub (MIU), and UIM and MIU related UBD (UMI) have a single helix as a Ub-binding site. yNpl4 CTD, MIU, and UMI but not UIM exhibit the same helix orientation relative to the bound Ub. Therefore, we compared the Ub-binding helices of yNpl4 CTD, RNF168 UMI (PDB 5XIS [https://doi.org/10.2210/pdb5xis/pdb]) ${ }^{38}$, and Rabex5 MIU (PDB 2C7N [https://doi.org/10.2210/pdb2c7n/ pdb] $)^{39}$ (Fig. 5b). These UBDs have an N-terminal hydrophilic residue for hydrogen bonding with the $\mathrm{NH}$ groups of Ala46 and Gly47 of Ub, and a central hydrophobic residue for the interaction with the hydrophobic pocket formed by Leu8, Ile44 and Val70 of Ub (Fig. 5b, c). The differences of the N-terminal hydrophilic residue and central hydrophobic residue in the Ub-binding helix affect its orientation relative to Ub. The central hydrophobic residues are Ile in Npl4 CTD and UMI and Ala in MIU. The longer side chain of Ile in yNp14 CTD or UMI pushes out the C-terminal end of the helical UBD, whereas those N-terminal hydrophilic residues move close proximity to the $\mathrm{NH}$ groups of Ala46 and Gly47 of Ub. 
a
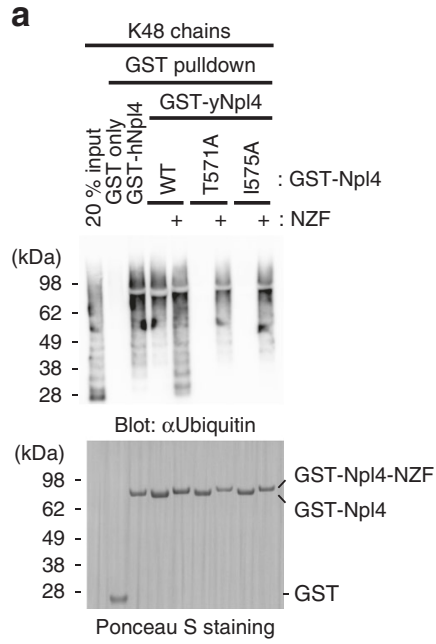

b
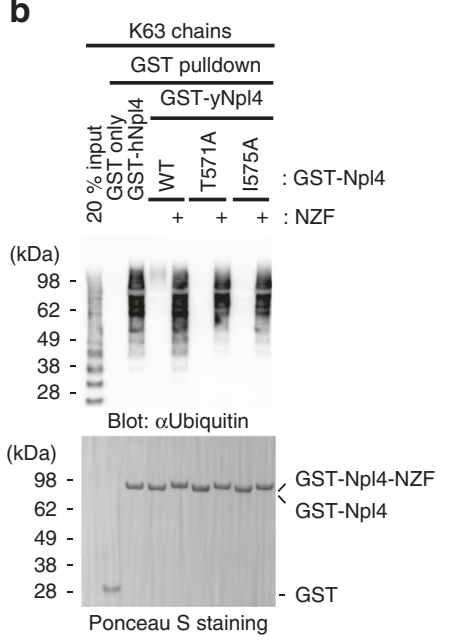

c

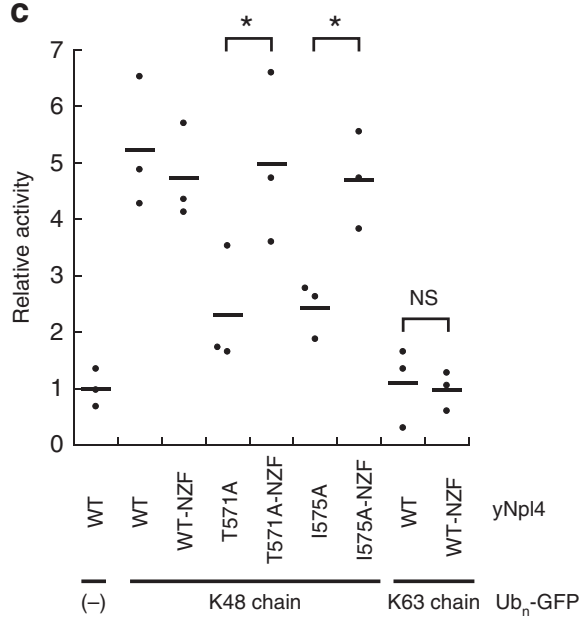

Fig. 4 Analysis of the NZF-fused yNp14. a, b Analysis of the binding of GST-hNpl4, GST-yNpl4 or GST-yNp|4-NZF to K48 chains (a) or K63 chains (b) by pulldown assays. The bound $\mathrm{Ub}$ chains were detected by immunoblotting with anti-Ub antibody (upper panel). Blot membranes were stained with Ponceau S (lower panel). $20 \%$ input means that $20 \%$ of the volume of the sample (Ub chains) that was mixed with GST-yNpl4- or GST-yNpl4-NZF-bound glutathione resin. These experiments were repeated with distinct samples (Supplementary Fig. 6c). Source data are provided as a Source Data file. c ATP hydrolysis rates of the Cdc48-UN complex containing yNpl4 or yNpl4-NZF with K48-Ub $b_{n}-$ GFP or K63-Ub ${ }_{n}$-GFP. The rates were normalized to the average of the ATP hydrolysis rates of the wild-type Cdc48-UN complex without $U b_{n}-G F P$. The line represents the mean of the rates after normalization (mean values; $n=3$ independent experiments; ${ }^{*} P<0.05$ from Student's $t$-test).

a

b

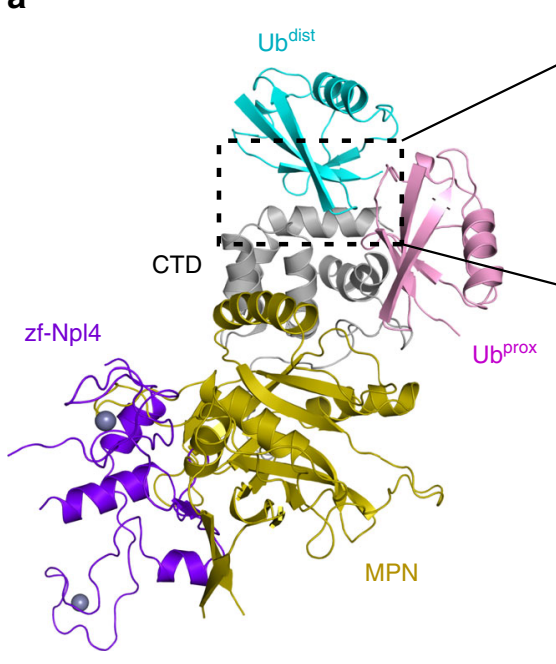

b
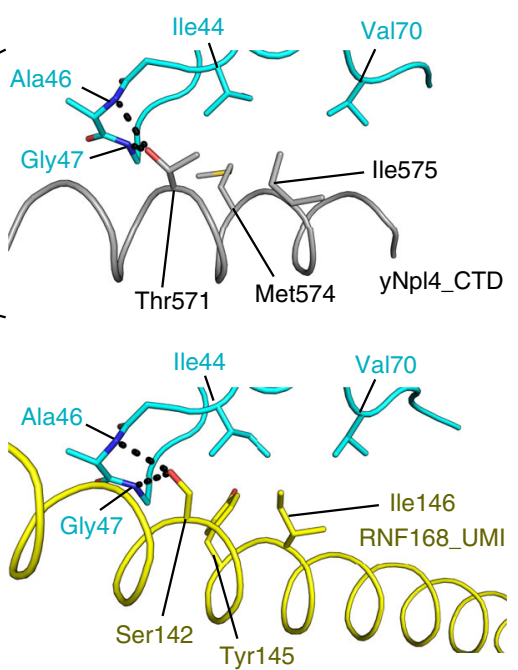

C
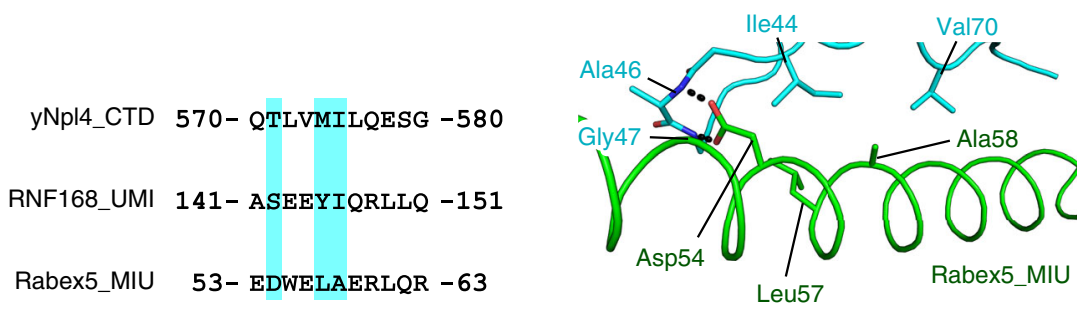

Fig. 5 Comparison of the C-terminal helix of yNpl4, RNF168 UMI, and Rabex-5 MIU. a Overall structure of the $y$ Npl4-K48-Ub 2 complex. The coloring scheme is the same as that in Fig. 2a. b Structural comparison of the Ub-binding interfaces of the C-terminal helix of yNpl4 (this study), RNF168 UMI (PDB 5XIS [https://doi.org/10.2210/pdb5xis/pdb]) ${ }^{38}$, and Rabex-5 MIU (PDB 2C7M [https://doi.org/10.2210/pdb2c7m/pdb])39. The coloring scheme of the yNpl4-Ubdist complex is the same as that in Fig. 2a. RNF168 UMI and Rabex-5 MIU are colored yellow and green, respectively. Hydrogen bonds are shown as black dotted lines. The residues involved in the binding between yNpl4 and Ubdist and the corresponding residues of RNF168 UMI and Rabex-5 MIU are shown as sticks. c Sequence alignment of the C-terminal helix of yNpl4, RNF168 UMI, and Rabex-5 MIU. The residues involved in the binding between yNpl4 and Ubdist and the corresponding residues of RNF168 UMI and Rabex-5 MIU are highlighted in cyan. 
Because of this proximity, the $\mathrm{N}$-terminal hydrophilic residue of yNpl4 CTD and UMI are Thr and Ser, respectively, which are shorter than Asp, the N-terminal hydrophilic residue of MIU.

The cryo-EM structure of the substrate-engaged Cdc48- $\mathrm{UN}^{22}$ contains two folded $\mathrm{Ub}$ moieties, which correspond to Ub ${ }^{\text {dist }}$ and Ubprox in the present crystal structure of yNp14 $113-580-\mathrm{K}^{13}-\mathrm{Ub}_{2}$ (Fig. 6a). In both structures, the C-terminal helix of yNpl4 CTD interacts with the Ile44-centered hydrophobic patch of Ub dist (Fig. 6b), and therefore, the Ub ${ }^{\text {dist }}$ recognition mode of $y N p l 4$ is essentially the same. On the other hand, the Ubprox recognition mode is different. In the $\mathrm{yNpl} 4-\mathrm{K} 48-\mathrm{Ub}_{2}$ structure, Ser498 of yNpl4 forms a hydrogen bond with Arg42 of Ubprox. GST pulldown and SPR analysis of the S498R mutant of yNpl4 showed that Ser498 of yNpl4 is actually involved in binding to K48 chains (Fig. 2d, Supplementary Fig. 3b and Table 2). In the structure of the substrate-engaged Cdc48-UN, Ub by $\sim 10 \AA$, as compared with yNpl4-K48- $\mathrm{Ub}_{2}$, and does not interact with Ser498 of yNpl4 (Fig. 6b). This Ubprox movement appears to be coupled with threading of the unfolded Ub moiety; i.e., Ubprox interacts with Ser498 of yNpl4 at the initial Ub chain recognition step. At the subsequent substrate-threading step, the unfolded Ub is inserted into the central pore of $\mathrm{Cdc} 48$, and pulls $\mathrm{Ub}^{\text {prox }}$, which loses its interaction with Ser498 of yNpl4.

We also determined the crystal structure of yNpl4 $4^{113-580_{-}}$ yUfd1288-305. We docked the structure of the NBM region of Ufd1 into the cryo-EM structure of the substrate-engaged Cdc48-UN ${ }^{22}$ (Fig. 6c). The present yUfd1 ${ }^{288-305}$ model was nicely fitted into the unassigned density observed in the cryo-EM map (Fig. 6c, d), which was supposed to correspond to a part of yUfd 122 . This indicates that the yNpl4-Ufd 1 interaction revealed by crystallography of the UN complex similarly occurs in the substrate-engaged Cdc48-UN (Fig. 6d).

Inhibitors of Ub-proteasome system (UPS) have recently been developed as anticancer drugs ${ }^{40}$. Because the p97/Cdc48-UN complex plays crucial roles upstream of the proteasome, its inhibition has emerged as a novel therapeutic target in cancer cells ${ }^{41}$. However, since p97/Cdc48 also plays various roles outside the UPS pathway, its inhibition may affect many cell functions. In the context, disruptions of the specific p97-cofactor association will likely lead to the novel p97 inhibitors with enhanced specificity of anticancer activity. Inhibition of the interaction between Ufd1 and Npl4 prevents formation of the Cdc48-UN complex (Fig. 3f). Our present structure of yNpl4 $4^{113-580}-\mathrm{yUfd} 1^{288-305}$ might facilitate the development of such anticancer drugs by serving as a useful platform for structure-based design.

\section{Methods}

Preparation of Npl4, Ufd1, and Cdc48. The codon-optimized cDNAs of yNpl4, hNpl4, and yUfd1 were synthesized (Eurofins) to improve their expressions in Escherichia coli. For crystallization, the gene encoding yNpl4 $4^{113-580}$ or yUfd1 $1^{288-305}$ was cloned into the pGEX-6P1 expression vector using BamHI and XhoI sites or pCold-GST expression vector using NdeI and XhoI sites, respectively, to produce the N-terminal GST fusion proteins. For GST pulldown assays, the fulllength $\mathrm{yNpl} 4$ and $y \mathrm{Ufd} 1$ genes were cloned into the pGEX-6P1 expression vector using BamHI and XhoI sites ${ }^{15}$, and the Cdc48 gene was cloned into the pET21a expression vector using BamHI and XhoI sites. For SPR analyses, the gene encoding yNpl4 ${ }^{113-580}$ or hNpl4 $4^{105-608}$ was cloned into the pET28a expression vector using $\mathrm{NdeI}$ and $\mathrm{XhoI}$ sites to produce the $\mathrm{N}$-terminal $\mathrm{His}_{6}$-tagged proteins. For fluorescence anisotropy-based affinity measurements, the GWCCPGCC sequence was attached to the C-terminus of yUfd $1^{288-305}$. The yUfd1 $1^{288-305}$. GWCCPGCC gene was cloned into the pCold-SUMO expression vector using NdeI and $\mathrm{XhoI}$ sites to produce the $\mathrm{N}$-terminal $\mathrm{His}_{6}$-tagged SUMO fusion protein. Mutations were generated by PCR-based mutagenesis. Primer sequences used in this study are shown in Supplementary Table 1. E. coli strain Rosetta (DE3) cells (Invitrogen) were transformed with each expression vector, and cultured in LB medium containing $100 \mathrm{mg} \mathrm{L}^{-1}$ ampicillin for the pGEX-6P1 or pCold expression vectors or $50 \mathrm{mg} \mathrm{L}^{-1}$ kanamycin for the pET28a expression vector at $37^{\circ} \mathrm{C}$. When the optical density of the culture at $600 \mathrm{~nm}$ reached $\sim 0.5$, isopropyl- $\beta$-D-thiogalactopyranoside (IPTG) was added to a final concentration of $0.1 \mathrm{mM}$ to induce protein expression, and the culture was further continued for $18 \mathrm{~h}$ at $20^{\circ} \mathrm{C}$ for the
pGEX-6P1 and pET28a expression vectors and for $24 \mathrm{~h}$ at $15^{\circ} \mathrm{C}$ for the pCold expression vector. The cells transformed with the pGEX-6P1 or pCold-GST expression vector were disrupted by sonication in phosphate buffered saline (PBS) containing $1 \mathrm{mM}$ dithiothreitol (DTT) and $0.5 \%$ Triton X-100, and purified by a Glutathione Sepharose FF column (GE Healthcare) and a Resource Q anion exchange column (GE Healthcare). The GST tag of GST-yNpl4 ${ }^{113-580}$ was cleaved by HRV3C protease, and the sample was further purified by a Resource Q anion exchange column and a HiLoad 16/60 Superdex 75 column (GE Healthcare) in $10 \mathrm{mM}$ Tris- $\mathrm{HCl}$ buffer ( $\mathrm{pH} 7.2$ ) containing $50 \mathrm{mM} \mathrm{NaCl}$ and $5 \mathrm{mM} \beta$ mercaptoethanol. To prepare the yNpl4 $4^{113-580}-\mathrm{yUfd}^{288-305}$ complex for crystallization, a two-fold molar excess of GST-yUfd $11^{288-305}$ was incubated at $4{ }^{\circ} \mathrm{C}$ for 10 min with yNpl4 ${ }^{113-580}$. The GST-yUfd1 $1^{288-305}-\mathrm{yNpl} 4^{113-580}$ complex was purified by a HiLoad 16/60 Superdex 75 column in $10 \mathrm{mM}$ Tris-HCl buffer (pH 7.2) containing $50 \mathrm{mM} \mathrm{NaCl}$ and $5 \mathrm{mM} \beta$-mercaptoethanol. The GST tag of GSTyUfd1 $1^{288-305}-\mathrm{yNpl}^{113}-580$ was cleaved by HRV3C protease. To remove GST, the sample was passed over a Glutathione Sepharose FF column pre-equilibrated with $10 \mathrm{mM}$ Tris-HCl buffer ( $\mathrm{pH} 7.2$ ) containing $50 \mathrm{mM} \mathrm{NaCl}$ and $5 \mathrm{mM} \beta$ mercaptoethanol. The cells transformed with the pCold-SUMO or pET28a expression vectors were disrupted by sonication in $50 \mathrm{mM}$ Tris- $\mathrm{HCl}$ buffer $(\mathrm{pH}$ 8.0) containing $150 \mathrm{mM} \mathrm{NaCl}$ and $0.5 \%$ Triton $\mathrm{X}-100$, and purified by a nickelnitrilotriacetic acid (Ni-NTA) column (Qiagen) and a Resource Q anion exchange column (GE Healthcare), except for Cdc48. For preparation of Cdc48, the Cdc48expressing cells were lysed in lysis buffer $(50 \mathrm{mM}$ sodium phosphate buffer $(\mathrm{pH}$ 7.0) containing $300 \mathrm{mM} \mathrm{NaCl}, 10 \%$ glycerol, $1 \mathrm{mM}$ Tris (2-carboxiehyl) phosphine hydrochloride (TCEP), $5 \mathrm{mM} \mathrm{MgCl} 2$, and $100 \mu \mathrm{M} \mathrm{ATP}$ ) and disrupted by sonication. After addition of Triton X-100 (final concentration, $0.1 \%$ ), the lysate was clarified by centrifugation at $29,300 \mathrm{x}$ g for $30 \mathrm{~min}$. The resultant supernatant was incubated with TALON resin (Takara). After extensive washing, Cdc48 was eluted in $50 \mathrm{mM}$ HEPES-NaOH buffer ( $\mathrm{pH} 7.1$ ) containing $100 \mathrm{mM} \mathrm{NaCl}, 0.3 \mathrm{M}$ imidazole, $5 \mathrm{mM} \mathrm{MgCl}_{2}, 100 \mu \mathrm{M}$ ATP, and $0.5 \mathrm{mM}$ TCEP. To further enrich for hexameric Cdc48, the solution was loaded on a Superose $610 / 300$ column equilibrated to $50 \mathrm{mM}$ HEPES-NaOH buffer ( $\mathrm{pH}$ 7.5) containing $100 \mathrm{mM} \mathrm{NaCl}, 5 \mathrm{mM}$ $\mathrm{MgCl}_{2}$, and $0.5 \mathrm{mM}$ TCEP with a flow rate of $0.25 \mathrm{~mL} \mathrm{~min}^{-1}$. To prepare the yUfd1-His 6 -yNpl4 ${ }^{113-580}$ complex for SPR analysis, the cells expressing GSTyUfd1 and those expressing $\mathrm{His}_{6}-\mathrm{yNpl}^{113-580}$ were mixed and disrupted at the same time by sonication in PBS containing $1 \mathrm{mM}$ DTT and $0.5 \%$ Triton X-100. The cleared lysate was loaded onto a Glutathione Sepharose FF column (GE Healthcare). The GST tag of GST-yUfd1-His ${ }_{6}-\mathrm{yNpl}^{113-580}$ was cleaved by HRV3C protease, and the sample was further purified by a Ni-NTA column (Qiagen) and a HiLoad 16/60 Superdex 75 column (GE Healthcare) in $10 \mathrm{mM}$ HEPES-NaOH (pH 7.5) containing $150 \mathrm{mM} \mathrm{NaCl}$.

Preparation of Ub chains and ubiquitylated substrates. Ub was overproduced in E. coli strain Rosetta (DE3) cells (Invitrogen) transformed with the pET26b expression vector harboring the Ub gene in $\mathrm{LB}$ medium containing $50 \mathrm{mg} \mathrm{L}^{-1}$ kanamycin at $20^{\circ} \mathrm{C}$. The SeMet-labeled Ub, Ub (P19M V26M), and Ub (I30M) was overproduced in the methionine-auxotroph E. coli strain B834 (DE3) cells in the customized medium equivalent to LeMaster medium (Code No. 06780, Nacalai tesque) with $30 \mu \mathrm{g} \mathrm{mL}^{-1} \mathrm{~L}$-SeMet (Nacalai tesque) and $50 \mathrm{mg} \mathrm{L}^{-1}$ kanamycin at $20^{\circ} \mathrm{C}$. The cells were disrupted by sonication in $50 \mathrm{mM}$ ammonium acetate buffer (pH 4.5). The cleared lysates of Ub and Ub variant were incubated for $5 \mathrm{~min}$ at 80 and $60^{\circ} \mathrm{C}$, respectively. The denatured and insolubilized E. coli proteins were pelleted by centrifugation at $30,000 \times g$ for $60 \mathrm{~min}$. The supernatant was purified by a Resource S cation exchange column (GE Healthcare) and a HiLoad 26/60 Superdex 75 size-exclusion column (GE Healthcare) in $10 \mathrm{mM}$ Tris- $\mathrm{HCl}$ buffer (pH 7.2) containing $50 \mathrm{mM} \mathrm{NaCl}$. The purified $\mathrm{Ub}$ was concentrated with an Amicon Ultra-15 10,000 MWCO filter (Millipore).

K48-, K63-, and M1- $\mathrm{Ub}_{4}$, and SeMet-labeled $\mathrm{K} 48-\mathrm{Ub}_{2}$ were synthesized enzymatically. For K48-Ub $\mathrm{Ub}_{4}$ synthesis, E1 $(0.25 \mu \mathrm{M}), \mathrm{E} 2-25 \mathrm{~K}(5 \mu \mathrm{M})$, and Ub $(2 \mathrm{mM})$ were mixed in the reaction buffer $(50 \mathrm{mM}$ Tris- $\mathrm{HCl}(\mathrm{pH} 9.0)$ containing $10 \mathrm{mM} \mathrm{ATP}, 10 \mathrm{mM} \mathrm{MgCl}_{2}$, and $0.6 \mathrm{mM} \mathrm{DTT}$ ) and incubated at $37^{\circ} \mathrm{C}$ for $15 \mathrm{~h}$. For SeMet-labeled K48-Ub 2 synthesis, E1 $(0.25 \mu \mathrm{M}), \mathrm{E} 2-25 \mathrm{~K}(5 \mu \mathrm{M})$, and Ub $(2 \mathrm{mM})$ were mixed in the reaction buffer and incubated at $37^{\circ} \mathrm{C}$ for $15 \mathrm{~h}$. For K63$\mathrm{Ub}_{4}$ synthesis, E1 $(0.25 \mu \mathrm{M})$, Ubc13 $(10 \mu \mathrm{M})$, MMS2 $(10 \mu \mathrm{M})$ and $\mathrm{Ub}(2 \mathrm{mM})$ were mixed in the reaction buffer and incubated at $37^{\circ} \mathrm{C}$ for $15 \mathrm{~h}$. For M1-Ub $\mathrm{U}_{4}$ synthesis E1 $(0.2 \mu \mathrm{M}), \mathrm{UbcH} 7(5 \mu \mathrm{M})$, HOIP (residues 697-1072; $0.1 \mu \mathrm{M})$ and Ub $(2 \mathrm{mM}$ ) were mixed in the reaction buffer and incubated at $37^{\circ} \mathrm{C}$ for $15 \mathrm{~h}$. Each reaction solution was mixed with four volumes of $50 \mathrm{mM}$ ammonium acetate buffer $(\mathrm{pH}$ 4.5) and loaded onto a Resource S cation exchange column (GE Healthcare) preequilibrated with $50 \mathrm{mM}$ ammonium acetate buffer ( $\mathrm{pH} 4.5$ ) containing $140 \mathrm{mM}$ $\mathrm{NaCl}$. The synthesized $\mathrm{Ub}_{4}$ species or SeMet-labeled $\mathrm{K} 48-\mathrm{Ub}_{2}$ were eluted with a linear gradient of $140-400 \mathrm{mM} \mathrm{NaCl}$ in $50 \mathrm{mM}$ ammonium acetate buffer ( $\mathrm{pH} 4.5)$ Peak fractions containing the $\mathrm{Ub}_{4}$ species or SeMet-labeled $\mathrm{K} 48-\mathrm{Ub}_{2}$ were loaded onto a HiLoad 16/60 Superdex 75 size-exclusion column (GE Healthcare) with $10 \mathrm{mM}$ HEPES-NaOH (pH 7.5) containing $150 \mathrm{mM} \mathrm{NaCl}$. The purified Ub chains were concentrated to $\sim 1 \mathrm{mM}$ and stored at $-80^{\circ} \mathrm{C}$ until use.

For preparation of $\mathrm{Ub}_{\mathrm{n}}$-GFP substrates, the gene encoding Ub-sfGFPcytochrome $b 2$ derived tail (Ub-GFP) ${ }^{42}$ was cloned into the pET21a expression vector using $N d e \mathrm{I}$ and $E c o R I$ sites. $\mathrm{Ub}_{\mathrm{n}}-\mathrm{GFP}$ substrates were enzymatically synthesized and purified by column chromatography ${ }^{14}$. For K48-Ub ${ }_{n}-G F P$ 

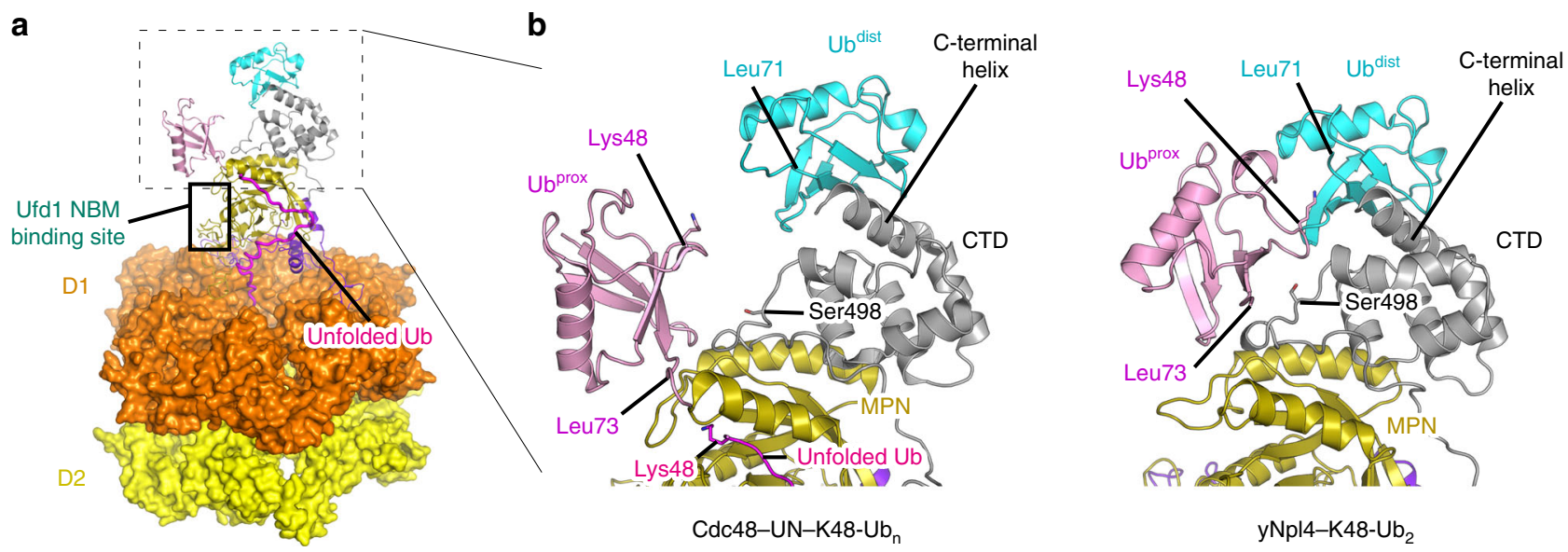

c

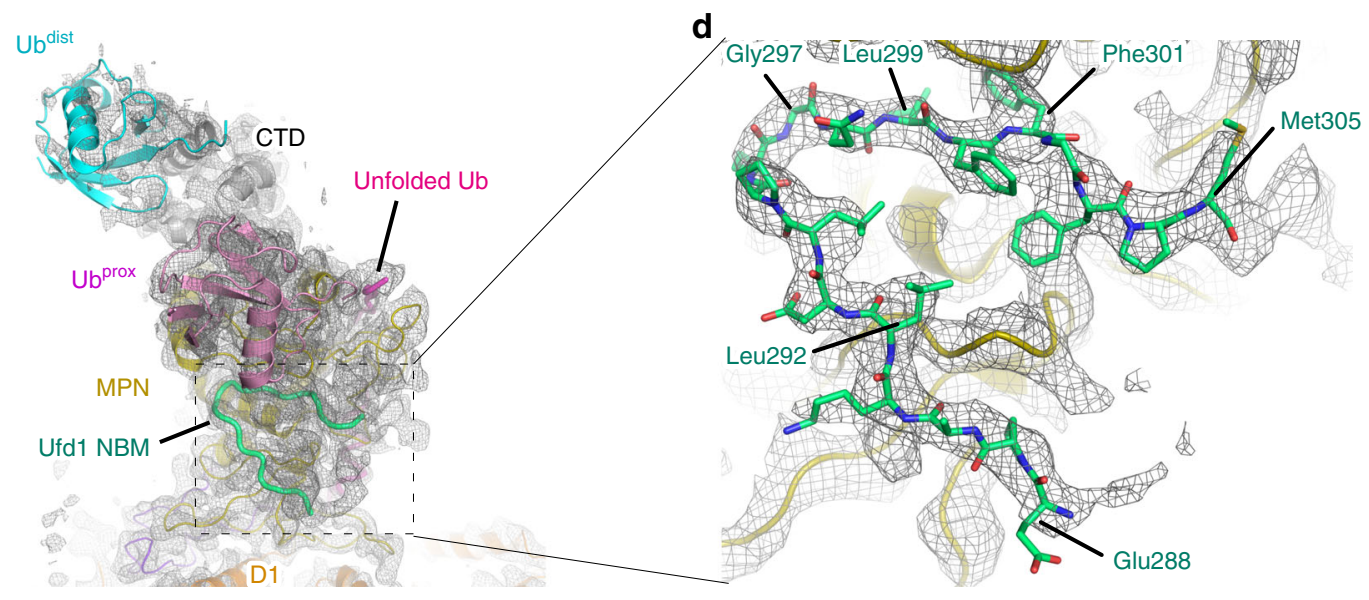

Fig. 6 Comparison of the structures of yNpl4-K48-Ub $\mathbf{b}_{2}$ yNpl4-yUdf1 and the substrate-engaged Cdc48-UN complex. a Overall view of the cryo-EM structure of the Cdc48-UN complex (PDB 6OA9 [https://doi.org/10.2210/pdb6oa9/pdb]) 22. The coloring scheme is the same as that in Fig. 1. The unfolded $\mathrm{Ub}$ is colored magenta. b Comparison of the K48 chain-binding site of yNpl4-K48- $\mathrm{Ub}_{2}$ and the substrate-engaged Cdc48-UN complex. The coloring scheme is the same as that in Fig. 6a. c, $\mathbf{d}$ The structure of yNpl4-yUfd1 was superposed on the substrate-engaged Cdc48-UN complex using the Coot LSQ protocol with the zf-NpI4-MPN-CTD structure of yNpl4 as the reference ${ }^{47}$. yUfd1 NBM was further fitted as a rigid body into the cryo-EM map of the substrate-engaged Cdc48-UN (EMD-0665) 22 and was refined against it with real space refinement in Phenix ${ }^{45}$. The coloring scheme is the same as that in Fig. 3a. The cryo-EM map is shown as grey mesh. c Overall view of yNpl4-yUfd1 and two folded Ub moieties with the cryo-EM map contoured at $5 \sigma$ level. d Close-up view of the interaction between yNpl4 and yUfd1 with the cryo-EM map contoured at $8 \sigma$ level.

synthesis, $10 \mu \mathrm{M}$ Ub-GFP, $1 \mu \mathrm{M}$ E1, $20 \mu \mathrm{M}$ gp78RING-Ube2g2, and $400 \mu \mathrm{M}$ Ub were incubated in $20 \mathrm{mM}$ HEPES-NaOH buffer ( $\mathrm{pH} 7.4$ ) containing $10 \mathrm{mM}$ ATP and $10 \mathrm{mM} \mathrm{MgCl}_{2}$ at $37^{\circ} \mathrm{C}$ overnight. For K63-Ub ${ }_{\mathrm{n}}-\mathrm{GFP}$ synthesis, $20 \mu \mathrm{M} \mathrm{Ubc13/}$ MMS2 was used, instead of $20 \mu \mathrm{M}$ gp78RING-Ube2g2. $\mathrm{Ub}_{\mathrm{n}}$-GFP substrates were bound to Ni-NTA beads, washed, and then eluted in $50 \mathrm{mM}$ HEPES-NaOH buffer (pH 7.5) containing $100 \mathrm{mM} \mathrm{NaCl}$ and $0.3 \mathrm{M}$ imidazole. The elution was loaded onto a Superdex 200 increase 10/300 column equilibrated to $50 \mathrm{mM}$ HEPES-NaOH (pH 7.5) buffer containing $100 \mathrm{mM} \mathrm{NaCl}$ and $10 \%$ glycerol. Fractions containing long $\mathrm{Ub}$ chains (approximately $\mathrm{Ub}_{10}$ in average) were collected for ATPase assay.

Crystallization. For crystallization of yNpl $4^{113-580}$ and the yNp1413-580-yUfd1 $1^{288-305}$ complex, the E123A, K124A, and E125A mutations were introduced into yNpl4 $4^{113-580}$ so as to reduce excess surface conformational entropy ${ }^{27}$. For crystallization of the yNp14 ${ }^{113-580}-$ SeMet-labeled K48-Ub 2 (WT, P19M V26M, or I30M) complex, yNpl4 $4^{113-580}$ was mixed with the SeMet-labeled $\mathrm{K} 48-\mathrm{Ub}_{2}$ in a molar ratio of 1:1.2. Initial crystallization screening was performed with the sitting drop vapor diffusion method at $20^{\circ} \mathrm{C}$ using a Mosquito liquid-handling robot (TTP Lab Tech). We tested about 500 conditions with crystallization reagent kits supplied by Hampton Research and Qiagen. Initial hits were further optimized. The best crystals of yNpl4 $4^{113-580}$ were grown at $20^{\circ} \mathrm{C}$ with the sitting drop vapor diffusion method by mixing $0.5 \mu \mathrm{L}$ of protein solution with an equal amount of reservoir solution containing $4 \%$ Tacsimate $(\mathrm{pH} 6.0$ ) and 12\% PEG3350 and equilibration against $500 \mu \mathrm{L}$ of the reservoir solution. The best crystals of the yNp1 $4^{113-580}$-yUfd $1^{288-305}$ complex were grown at $20^{\circ} \mathrm{C}$ with the sitting drop vapor diffusion method by mixing $0.2 \mu \mathrm{L}$ of protein solution with an equal amount of reservoir solution containing $90 \mathrm{mM}$ Bis-Tris- $\mathrm{HCl}$ buffer ( $\mathrm{pH}$ 7.5), $19 \%$ PEG3350, and $10 \mathrm{mM}$ ATP and equilibration against $50 \mu \mathrm{L}$ of the reservoir solution. The best crystals of the yNpl4 ${ }^{113-580}$-SeMet-labeled K48-Ub 2 (WT) complex were grown at $20^{\circ} \mathrm{C}$ with the sitting drop vapor diffusion method by mixing $0.5 \mu \mathrm{L}$ of protein solution with an equal amount of $100 \mathrm{mM}$ Bicine- $\mathrm{NaOH}$ buffer ( $\mathrm{pH}$ 9.0) containing 34\% PEG3350 and $200 \mathrm{mM} \mathrm{Li}_{2} \mathrm{SO}_{4}$ and equilibration against $500 \mu \mathrm{L}$ of reservoir solution containing $100 \mathrm{mM}$ Bicine- $\mathrm{NaOH}$ buffer $(\mathrm{pH}$ 9.0), $18 \%$ PEG3350, and $200 \mathrm{mM} \mathrm{Li}_{2} \mathrm{SO}_{4}$. The best crystals of the yNpl4 $4^{113-580}-$ SeMet-labeled $\mathrm{K} 48-\mathrm{Ub}_{2}\left(\mathrm{P} 19 \mathrm{M}\right.$ V26M) complex were grown at $20^{\circ} \mathrm{C}$ with the sitting drop vapor diffusion method by mixing $0.5 \mu \mathrm{L}$ of protein solution with an equal amount of $100 \mathrm{mM}$ Bicine- $\mathrm{NaOH}$ buffer ( $\mathrm{pH} 9.0$ ) containing $24 \%$ PEG3350, $200 \mathrm{mM} \mathrm{NaCl}$, 3\% 1,5-diaminopentane, and $10 \mathrm{mM} \mathrm{MgCl}_{2}$ and equilibration against $500 \mu \mathrm{L}$ of reservoir solution containing $100 \mathrm{mM}$ Bicine- $\mathrm{NaOH}$ buffer ( $\mathrm{pH} 9.0$ ), 20\% PEG3350, and $200 \mathrm{mM} \mathrm{NaCl}$. The best crystals of the yNp14 $113-580-$ SeMet-labeled $\mathrm{K} 48-\mathrm{Ub}_{2}(\mathrm{I} 30 \mathrm{M})$ complex were grown at $20^{\circ} \mathrm{C}$ with the sitting drop vapor diffusion method by mixing $0.5 \mu \mathrm{L}$ of protein solution with an equal amount of $100 \mathrm{mM}$ Bicine- $\mathrm{NaOH}$ buffer ( $\mathrm{pH} 9.0$ ) containing $23 \%$ PEG3350, $200 \mathrm{mM} \mathrm{Li}_{2} \mathrm{SO}_{4}, 3 \%$ 1,5-diaminopentane, and $10 \mathrm{mM} \mathrm{MgCl}_{2}$ and equilibration against $500 \mu \mathrm{L}$ of reservoir solution containing $100 \mathrm{mM}$ Bicine$\mathrm{NaOH}$ buffer (pH 9.0), 12\% PEG3350, $200 \mathrm{mM} \mathrm{Li}_{2} \mathrm{SO}_{4}$, and $10 \mathrm{mM} \mathrm{MgCl}_{2}$. For data collection, the crystals were transferred to cryostabilizing solution, which was the individual reservoir solution containing $30 \%$ glycerol for $\mathrm{yNp1} 4^{113-580}, 20 \%$ glycerol for yNpl4 $4^{113-580}-\mathrm{yUfd} 1^{288-305}$, or saturated trehalose for $\mathrm{yNp} 14^{113-580}-$ SeMet-labeled $\mathrm{K} 48-\mathrm{Ub}_{2}$ (WT, P19M V26M, or I30M). The cryoprotected crystals were flash frozen in liquid $\mathrm{N}_{2}$.

Structure determination. Diffraction data sets were collected at beamline BL41XU in SPring-8 (Hyogo, Japan) at $100 \mathrm{~K}$. PILATUS3 $6 \mathrm{M}$ (Dectris) was used for the data collection of yNpl4 ${ }^{113-580}$ and yNpl4 ${ }^{113-580}-$ SeMet-labeled $\mathrm{K}^{4} 8-\mathrm{Ub}_{2}$ and 
EIGER X $16 \mathrm{M}$ (Dectris) was used for the data collection of yNpl4 $4^{113-580}-y U f d 1^{288-305}$. The wavelengths for the data collection were 1.28260 , 0.97904 , and $1.00000 \AA$ for $\mathrm{yNpl} 4^{113-580}$, yNpl $4^{113-580}$-SeMet-labeled $\mathrm{K} 48-\mathrm{Ub}_{2}$, and $y N p 14^{113-580}-\mathrm{yUfd} 1^{288-305}$, respectively. The data sets were processed with HKL $2000^{43}$ and the CCP4 program suite ${ }^{44}$. To solve the structure of yNpl4 $113-580$ from the SAD data set using zinc anomalous scattering, the program Phenix was used for heavy-atom search, phase calculation, and density modification ${ }^{45}$. The structures of the yNpl4 $4^{113-580}-\mathrm{yUfd} 1^{288-305}$ and yNpl4 ${ }^{113-580}$-SeMet-labeled K48$\mathrm{Ub}_{2}$ complexes were determined by the molecular replacement method using the program MolRep ${ }^{46}$. The crystal structure of yNpl4113-580 was used as the search model. The solution of Ub moieties in the $\mathrm{yNpl4}-\mathrm{K} 48-\mathrm{Ub}_{2}$ structure was not found using the program MolRep with the crystal structure of Ub (PDB 1UBQ [https:// doi.org/10.2210/pdblubq/pdb] $)^{28}$ as the search model. We manually assigned the $\mathrm{Ub}$ models in residual density using the program $\mathrm{Coot}^{47}$. The position of $\mathrm{K} 48-\mathrm{Ub}_{2}$ was confirmed by the anomalous difference Fourier map of selenium atoms in the yNpl4-K48-Ub 2 (P19M V26M) and yNpl4-K48-Ub $(\mathrm{I} 30 \mathrm{M})$ structures (Supplementary Fig. 1c). The atomic models were corrected using $\operatorname{Coot}^{47}$ with careful inspection. Refinement was carried out using Phenix with iterative correction and refinement of the atomic models. There is one copy of the structure in the asymmetric unit of the yNpl4 ${ }^{113-580}$ or yNpl4 $4^{113-580}-\mathrm{yUfd}^{288-305}$ crystal. On the other hand, there are two yNpl4 $4^{113-580}$ and two Ub (Ub ${ }^{\text {dist }}$ and Ubrox) molecules in the asymmetric unit of the $y \mathrm{Npl}^{113-580}$-SeMet-labeled $\mathrm{K} 48-\mathrm{Ub}_{2}$ crystal. Torsion-angle NCS restrains were applied during refinement of the yNpl4-SeMetlabeled $\mathrm{K} 48-\mathrm{Ub}_{2}$ structure. The final models of $\mathrm{yNp} 4^{113-580}$,

$\mathrm{yNpl}^{113-580}-\mathrm{yUfd} 1^{288-305}$, and yNpl4 $4^{113-580}$-SeMet-labeled $\mathrm{K} 48-\mathrm{Ub}_{2}$ have excellent stereochemistry (Table 1). All molecular graphics were prepared with PyMOL (DeLano Scientific; http://www.pymol.org).

Sequence alignment. Multiple sequence alignment was performed using the program ClustalW ${ }^{48}$. The figure of the sequence alignment between $\mathrm{yNpl} 4$ and $\mathrm{hNpl} 4$ was prepared using the program ESPript $3^{49}$.

SPR analysis. SPR analysis was performed using Biacore T200 (GE healthcare) at $25^{\circ} \mathrm{C}$. Wild-type or mutant $\mathrm{His}_{6}-\mathrm{yNpl}^{113-580}$, yUfd1-His $6_{6}-\mathrm{yNpl} 4^{113-580}$, or $\mathrm{His}_{6}{ }^{-}$ hNpl $4^{105-600}$ was immobilized on a CM5 sensor chip by the amine-coupling method in $10 \mathrm{mM}$ HEPES-NaOH (pH 7.5) containing $150 \mathrm{mM} \mathrm{NaCl}$ and $0.05 \%$ Tween-20. The amount of the immobilized ligand for each experiment is shown in response units (RU) in Supplementary Fig. 7. $\mathrm{Ub}_{2}$ or $\mathrm{Ub}_{4}$ was prepared in a twofold serial dilution series and each dilution sample was injected for $60 \mathrm{~s}$ at a flow rate of $10 \mu \mathrm{L}$ per min in $10 \mathrm{mM}$ HEPES-NaOH buffer ( $\mathrm{pH} 7.5$ ) containing $150 \mathrm{mM}$ $\mathrm{NaCl}, 0.05 \%$ Tween-20, and $0.5 \mathrm{~g} \mathrm{~L}^{-1} \mathrm{BSA}$. The ranges of the concentrations of $\mathrm{Ub}_{2}$ or $\mathrm{Ub}_{4}$ were shown in Supplementary Fig. 7. Equilibrium dissociation constants $\left(K_{\mathrm{d}}\right)$ were calculated using Biacore T200 software. Data are shown as means \pm standard deviation from three independent experiments for each sample.

Fluorescence anisotropy-based affinity measurement. The purified $\mathrm{His}_{6}$-SUMOyUfd1 $288-305$-GWCCPGCC was labeled with an equimolar ratio of FlAsH-EDT2 (Santa Cruz) in $50 \mathrm{mM}$ Tris-HCl buffer ( $\mathrm{pH} 8.0$ ) containing $0.1 \% \beta$-mercaptoethanol at room temperature for $1 \mathrm{~h}$. Unreacted FlAsH-EDT2 was removed with PD-10 desalting columns (GE Healthcare). To measure binding affinities $\left(K_{\mathrm{d}}\right)$ of yNpl4113-580 for $y U f d 1^{288-305}, 10 \mu \mathrm{L}$ of $1 \mathrm{nM}$ FlAsH-labeled His ${ }_{6}$-SUMO-yUfd $1^{280-305}$ was aliquoted into a 384 -well black, low volume, and round bottom plate (Corning). $\mathrm{His}_{6}$ yNpl4 ${ }^{113-580}$ was prepared in a two-fold serial dilution series. Ten microliter of each diluted sample was added to wells containing FlAsH-labeled $\mathrm{His}_{6}$-SUMO-

yUfd $1^{280-305}$. Fluorescence polarization was recorded by an EnVision plate reader (PerkinElmer) using $\lambda_{\mathrm{ex}}=480 \mathrm{~nm}$ and $\lambda_{\mathrm{em}}=535 \mathrm{~nm}$ at $25^{\circ} \mathrm{C}$. $K_{\mathrm{d}}$ was calculated with the program KaleidaGraph (HULINKS) (Supplementary Fig. 8). The measurement was carried out three times for each sample in $50 \mathrm{mM}$ Tris- $\mathrm{HCl}$ buffer ( $\mathrm{pH}$ 8.0) containing $100 \mathrm{mM} \mathrm{NaCl}, 5 \mathrm{mM} \beta$-mercaptoethanol, and $0.1 \mathrm{~g} \mathrm{~L}^{-1} \mathrm{BSA}$.

Yeast strains and media. S. cerevisiae strains used in this study are listed in Supplementary Table 2. All strains are isogenic to W303. Unless otherwise stated, we used exponentially growing yeast cells cultured at $25^{\circ} \mathrm{C}$ in YPD medium (1\% yeast extract, $2 \%$ peptone, $2 \%$ glucose, $400 \mathrm{mg} \mathrm{L}^{-1}$ adenine sulfate, and $10 \mathrm{mg} \mathrm{L}^{-1}$ uracil) below an $\mathrm{OD}_{600}$ of $0.8\left(\mathrm{OD}_{600}\right.$ of 1 contained $3.80 \times 10^{7}$ cells $\mathrm{mL}^{-1}$ in our culture).

Immunoblotting of yeast total cell lysate. Cells corresponding to $1 \mathrm{OD}_{600}$ were harvested and extracted by the mild-alkali method ${ }^{50}$. Proteins were separated by SDS-PAGE on $4-12 \%$ NuPAGE Bis-Tris gels (Life Technologies) with MES buffer (Thermo Fisher Scientific) and transferred to PVDF membrane (GE Healthcare) on an XCell II Blot Module (Life Technologies). Immunoblotting was performed with the following antibodies: rabbit monoclonal antibody against K48 chains (Apu2; used at 1:1000 for immunoblotting; Millipore, Cat\#05-1307), mouse monoclonal antibody against the FLAG-tag (M2, HRP-conjugated; 1:2000; Sigma-Aldrich, Cat \#A8592), rabbit polyclonal antibody against Cdc48 (1:1000; gift from Dr. Kimura) and mouse monoclonal antibody against Pgk1 (22C5D8; 1:1000; Life Technologies, Cat\#459250). HRP-conjugated goat anti-mouse or -rabbit Ig (1:10,000), used as a secondary antibody, was purchased from Jackson ImmunoResearch Laboratories (Cat\#315-035-048 or Cat\# 111-035-144). Immunoblots were developed using ECL
Prime Western Blotting Detection Reagent (GE Healthcare) and analyzed on ImageQuant LAS4000 (GE Healthcare).

Yeast plate test. Yeast cells were grown in SC medium $(0.67 \%$ yeast nitrogen base without amino acids, $0.5 \%$ casamino acids, $2 \%$ glucose, $10 \mathrm{mM}$ potassium phosphate (pH 7.5), $400 \mathrm{mg} \mathrm{L}^{-1}$ adenine sulfate, $10 \mathrm{mg} \mathrm{L}^{-1}$ uracil, and $20 \mathrm{mg} \mathrm{L}^{-1}$ tryptophan) at $25^{\circ} \mathrm{C}$. Then serial dilutions (1:5) of overnight cultures were spotted onto SC agar plates.

ATPase assay. Solution $(40 \mu \mathrm{L})$ containing $150 \mathrm{nM}$ Cdc48, $150 \mathrm{nM}$ UN heterodimer and $150 \mathrm{nM} \mathrm{Ub}_{\mathrm{n}}$-GFP substrate in reaction buffer $(50 \mathrm{mM}$ HEPES-NaOH (pH 7.5), $50 \mathrm{mM} \mathrm{NaCl}, 10 \mathrm{mM} \mathrm{MgCl}_{2}, 0.5 \mathrm{mM}$ TCEP, $0.1 \mathrm{mg} \mathrm{mL}^{-1} \mathrm{BSA}$ ) were incubated at $37^{\circ} \mathrm{C}$ for $15 \mathrm{~min}$. To this, $10 \mu \mathrm{L}$ of $1 \mathrm{mM}$ ATP solution and $50 \mu \mathrm{L}$ of BIOMOL Green were added. Absorbance $(600 \mathrm{~nm})$ was measured at $5 \mathrm{~s}$ intervals in Enspire2300 (Perkin Elmer). The slope of the initial (0 to $50 \mathrm{~s}$ ), linear portion of the curve was used to calculate the rate. The data were normalized to the average of the ATP hydrolysis rates of the wild-type Cdc48-UN complex without substrate.

GST pulldown assay. For analysis of the Cdc48 complex formation, GST-Npl4 or GST-Ufd $1(0.5 \mu \mathrm{M})$ was immobilized on $5 \mu \mathrm{L}$ of Glutathione Sepharose $4 \mathrm{~B}$ beads (GE Hearthcare) and incubated for $1 \mathrm{~h}$ at room temperature with $0.5 \mu \mathrm{M}$ binding partner (untagged Ufd1 or $\mathrm{His}_{6}$-tagged Npl4) and $3 \mu \mathrm{M} \mathrm{Cdc48}(0.5 \mu \mathrm{M}$ hexamer) in $100 \mu \mathrm{L}$ of $50 \mathrm{mM}$ Tris- $\mathrm{HCl}(\mathrm{pH} \mathrm{7.5)}$ buffer containing $100 \mathrm{mM} \mathrm{NaCl}, 10 \%$ glycerol, and $0.1 \%$ Triton X-100 (binding buffer). For analysis of Ub chain binding, $1 \mu \mathrm{g}$ of polyUb chains were added in $100 \mu \mathrm{L}$ of the binding buffer. For analysis of Ub chain binding with the increasing concentrations of yUfd $1,0.5 \mu \mathrm{g}$ of polyUb chains and $0,0.25,0.5,1,2$, or $4 \mu \mathrm{M}$ yUfd 1 were added in $50 \mu \mathrm{L}$ of the binding buffer. After three washes with the binding buffer, the bound proteins were eluted with NuPAGE LDS sample buffer (Thermo Fisher Scientific) for $10 \mathrm{~min}$ at $70{ }^{\circ} \mathrm{C}$. The eluted proteins were separated by SDS-PAGE on 4-12\% NuPAGE Bis-Tris gels and visualized with Bio-Safe Coomassie Stain (Bio-Rad). Immunoblots were performed as described above with mouse monoclonal antibody against Ub (P4D1, HRP-conjugated; 1:500; Santa Cruz Biotechnology \# sc-8017 HRP).

Immunoprecipitation of 3xFLAG-tagged NPL4 proteins. Cell lysis and immunoprecipitation were performed essentially as previously described ${ }^{15}$. For antiFLAG immunoprecipitation, $5 \mu \mathrm{L}$ of anti-DDDDK-tag mAb-Magnetic beads (MBL M185-11) were used to precipitate FLAG-tagged protein complex from $1 \mathrm{mg}$ of cell lysate by incubating for $1 \mathrm{~h}$ at $4{ }^{\circ} \mathrm{C}$. After three washes with buffer A (50 mM Tris$\mathrm{HCl}$ (pH 7.5), $100 \mathrm{mM} \mathrm{NaCl}, 10 \%$ glycerol, $10 \mu \mathrm{M}$ bortezomib (LC Laboratories), $10 \mathrm{mM}$ iodoacetamide, and 1x Complete protease inhibitor cocktail (EDTA free; Roche)) containing $1 \%$ Triton X-100, the bound proteins were eluted with $1 \mathrm{X}$ NuPAGE LDS sample buffer for $10 \mathrm{~min}$ at $70{ }^{\circ} \mathrm{C}$.

Reporting summary. Further information on research design is available in the Nature Research Reporting Summary linked to this article.

\section{Data availability}

The coordinates and structure factors of yNpl4, yNpl4-K48- $\mathrm{Ub}_{2}$, and yNpl4-yUfdl have been deposited in the Protein Data Bank under the accession codes 6JWH [https://doi. org/10.2210/pdb6jwh/pdb], 6JWI [https://doi.org/10.2210/pdb6jwi/pdb], and 6JWJ [https://doi.org/10.2210/pdb6jwj/pdb], respectively. The uncropped gel and blot images for Figs. 2d, 3e-f, and 4a-b and Supplementary Figs. 1d, 3a-d, 5a-e, and $6 \mathrm{c}$ are provided as a Source Data file. Other data are available from the corresponding authors upon reasonable request.

Received: 18 May 2019; Accepted: 21 November 2019; Published online: 13 December 2019

\section{References}

1. Pickart, C. M. Ubiquitin in chains. Trends Biochem Sci. 25, 544-548 (2000).

2. Finley, D. Recognition and processing of ubiquitin-protein conjugates by the proteasome. Annu Rev. Biochem 78, 477-513 (2009).

3. Kwon, Y. T. \& Ciechanover, A. The ubiquitin code in the ubiquitinproteasome system and autophagy. Trends Biochem Sci. 42, 873-886 (2017)

4. Husnjak, K. \& Dikic, I. Ubiquitin-binding proteins: decoders of ubiquitinmediated cellular functions. Annu Rev. Biochem 81, 291-322 (2012).

5. Komander, D. \& Rape, M. The ubiquitin code. Annu Rev. Biochem 81, 203-229 (2012).

6. Meyer, H., Bug, M. \& Bremer, S. Emerging functions of the VCP/p97 AAAATPase in the ubiquitin system. Nat. Cell Biol. 14, 117-123 (2012).

7. Olszewski, M. M., Williams, C., Dong, K. C. \& Martin, A. The Cdc48 unfoldase prepares well-folded protein substrates for degradation by the $26 \mathrm{~S}$ proteasome. Commun. Biol. 2, 29 (2019). 
8. Saeki, Y. Ubiquitin recognition by the proteasome. J. Biochem 161, 113-124 (2017).

9. Stach, L. \& Freemont, P. S. The AAA+ ATPase p97, a cellular multitool. Biochem J. 474, 2953-2976 (2017).

10. Bodnar, N. \& Rapoport, T. Toward an understanding of the Cdc48/p97 ATPase. F1000Res 6, 1318 (2017).

11. van den Boom, J. \& Meyer, H. VCP/p97-Mediated Unfolding as a Principle in Protein Homeostasis and Signaling. Mol. Cell 69, 182-194 (2018).

12. Meyer, H. H., Shorter, J. G., Seemann, J., Pappin, D. \& Warren, G. A complex of mammalian ufd1 and npl4 links the AAA-ATPase, p97, to ubiquitin and nuclear transport pathways. EMBO J 19, 2181-2192 (2000).

13. Bodnar, N. O. \& Rapoport, T. A. Molecular mechanism of substrate processing by the Cdc48 ATPase Complex. Cell 169, 722-735 e9 (2017).

14. Blythe, E. E., Olson, K. C., Chau, V. \& Deshaies, R. J. Ubiquitin- and ATPdependent unfoldase activity of P97/VCP.NPLOC4.UFD1L is enhanced by a mutation that causes multisystem proteinopathy. Proc. Natl Acad. Sci. USA 114, E4380-E4388 (2017).

15. Tsuchiya, $H$. et al. In vivo ubiquitin linkage-type analysis reveals that the Cdc48-Rad23/Dsk2 axis contributes to K48-linked chain specificity of the proteasome. Mol. Cell 66, 488-502 e7 (2017).

16. DeLaBarre, B. \& Brunger, A. T. Complete structure of p97/valosin-containing protein reveals communication between nucleotide domains. Nat. Struct. Biol. 10, 856-863 (2003).

17. Tang, W. K. et al. A novel ATP-dependent conformation in p97 N-D1 fragment revealed by crystal structures of disease-related mutants. EMBO J. 29, 2217-2229 (2010).

18. Banerjee, S. et al. $2.3 \AA$ resolution cryo-EM structure of human p97 and mechanism of allosteric inhibition. Science 351, 871-875 (2016).

19. Bodnar, N. O. et al. Structure of the Cdc48 ATPase with its ubiquitin-binding cofactor Ufd1-Npl4. Nat. Struct. Mol. Biol. 25, 616-622 (2018).

20. Briggs, L. C. et al. Analysis of nucleotide binding to $\mathrm{P} 97$ reveals the properties of a tandem AAA hexameric ATPase. J. Biol. Chem. 283, 13745-13752 (2008)

21. Xia, D., Tang, W. K. \& Ye, Y. Structure and function of the AAA+ ATPase p97/Cdc48p. Gene 583, 64-77 (2016)

22. Twomey, E. C. et al. Substrate processing by the Cdc48 ATPase complex is initiated by ubiquitin unfolding. Science 365, eaax1033 (2019).

23. Meyer, H. H., Wang, Y. \& Warren, G. Direct binding of ubiquitin conjugates by the mammalian p97 adaptor complexes, p47 and Ufd1-Npl4. EMBO J 21, 5645-5652 (2002).

24. Ye, Y., Meyer, H. H. \& Rapoport, T. A. Function of the p97-Ufd1-Npl4 complex in retrotranslocation from the ER to the cytosol: dual recognition of nonubiquitinated polypeptide segments and polyubiquitin chains. J. Cell Biol. 162, 71-84 (2003).

25. Worden, E. J., Dong, K. C. \& Martin, A. An AAA motor-driven mechanical switch in Rpn11 controls deubiquitination at the 26S proteasome. Mol. Cell 67, 799-811 e8 (2017).

26. Sato, Y. et al. Structural basis for specific cleavage of Lys 63-linked polyubiquitin chains. Nature 455, 358-362 (2008).

27. Goldschmidt, L., Cooper, D. R., Derewenda, Z. S. \& Eisenberg, D. Toward rational protein crystallization: a web server for the design of crystallizable protein variants. Protein Sci. 16, 1569-1576 (2007).

28. Vijay-Kumar, S., Bugg, C. E. \& Cook, W. J. Structure of ubiquitin refined at 1.8 Å resolution. J. Mol. Biol. 194, 531-544 (1987).

29. Bekes, M. et al. Recognition of Lys48-Linked Di-ubiquitin and Deubiquitinating activities of the SARS coronavirus papain-like protease. Mol. Cell 62, 572-585 (2016).

30. Rahighi, S. et al. Selective binding of AIRAPL tandem UIMs to Lys48-Linked Tri-ubiquitin chains. Structure 24, 412-422 (2016).

31. Kristariyanto, Y. A., Abdul Rehman, S. A., Weidlich, S., Knebel, A. \& Kulathu, Y. A single MIU motif of MINDY-1 recognizes K48-linked polyubiquitin chains. EMBO Rep. 18, 392-402 (2017).

32. Kulathu, Y., Akutsu, M., Bremm, A., Hofmann, K. \& Komander, D. Two-sided ubiquitin binding explains specificity of the TAB2 NZF domain. Nat. Struct. Mol. Biol. 16, 1328-1330 (2009).

33. Ren, X. \& Hurley, J. H. VHS domains of ESCRT-0 cooperate in high-avidity binding to polyubiquitinated cargo. EMBO J. 29, 1045-1054 (2010).

34. Park, S., Isaacson, R., Kim, H. T., Silver, P. A. \& Wagner, G. Ufd1 exhibits the AAA-ATPase fold with two distinct ubiquitin interaction sites. Structure 13, 995-1005 (2005).

35. Bruderer, R. M., Brasseur, C. \& Meyer, H. H. The AAA ATPase p97/VCP interacts with its alternative co-factors, Ufd1-Npl4 and p47, through a common bipartite binding mechanism. J. Biol. Chem. 279, 49609-49616 (2004).

36. Davis, I. W. et al. MolProbity: all-atom contacts and structure validation for proteins and nucleic acids. Nucleic Acids Res 35, W375-W383 (2007).

37. Fuchs, A. C. D., Maldoner, L., Wojtynek, M., Hartmann, M. D. \& Martin, J. Rpn11-mediated ubiquitin processing in an ancestral archaeal ubiquitination system. Nat. Commun. 9, 2696 (2018).
38. Takahashi, T. S. et al. Structural insights into two distinct binding modules for Lys63-linked polyubiquitin chains in RNF168. Nat. Commun. 9, 170 (2018).

39. Penengo, L. et al. Crystal structure of the ubiquitin binding domains of rabex5 reveals two modes of interaction with ubiquitin. Cell 124, 1183-1195 (2006).

40. Adams, J. The development of proteasome inhibitors as anticancer drugs. Cancer Cell 5, 417-421 (2004).

41. Vekaria, P. H., Home, T., Weir, S., Schoenen, F. J. \& Rao, R. Targeting p97 to Disrupt Protein Homeostasis in Cancer. Front Oncol. 6, 181 (2016).

42. Martinez-Fonts, K. \& Matouschek, A. A rapid and versatile method for generating proteins with defined ubiquitin chains. Biochemistry 55, 1898-1908 (2016).

43. Otwinowski, Z. \& Minor, W. Processing of X-ray diffraction data collected in oscillation mode. Methods Enzymol. 276, 307-326 (1997).

44. Collaborative Computational Project, N. The CCP4 Suite: programs for protein Crystallography. Acta Crystallogr D. Biol. Crystallogr 50, 760-763 (1994).

45. Adams, P. D. et al. PHENIX: a comprehensive Python-based system for macromolecular structure solution. Acta Crystallog. D Biol. Crystallogr. 66, (213-221 (2010).

46. Vagin, A. \& Teplyakov, A. MOLREP: an automated program for molecular replacement. J. Appl. Cryst. 30, 1022-1025 (1997).

47. Emsley, P. \& Cowtan, K. Coot: model-building tools for molecular graphics. Acta Crystallogr D. Biol. Crystallogr 60, 2126-2132 (2004).

48. Larkin, M. A. et al. Clustal W and Clustal X version 2.0. Bioinformatics 23, 2947-2948 (2007).

49. Robert, X. \& Gouet, P. Deciphering key features in protein structures with the new ENDscript server. Nucleic Acids Res 42, W320-W324 (2014).

50. Kushnirov, V. V. Rapid and reliable protein extraction from yeast. Yeast $\mathbf{1 6}$ 857-860 (2000).

\section{Acknowledgements}

We thank the beamline staff of the biological crystallography beamlines of BL41XU of SPring-8 (Hyogo, Japan) for technical help during data collection. This work was supported by JSPS/MEXT KAKENHI (JP16H04750 to Y. Sato, JP18K14913 to H.T., JP18H05498 to Y. Saeki, and JP18H05501 to S.F.), and Takeda Science Foundation (to Y. Saeki and K.T.)

\section{Author contributions}

The study was conceived by Y. Sato, Y. Saeki and S.F.Y. Sato carried out biophysical experiments and structure determination and wrote the paper. H.T. and Y. Saeki performed yeast experiments. A.Y. and K.O. assisted with the structure determination. K.T supervised the yeast experiments. All authors discussed the results. S.F. and Y. Saeki supervised the work, designed the experiments and wrote the paper.

\section{Competing interests}

The authors declare no competing interests.

\section{Additional information}

Supplementary information is available for this paper at https://doi.org/10.1038/s41467 019-13697-y.

Correspondence and requests for materials should be addressed to Y.S. or S.F.

Peer review information Nature Communications thanks Yogesh Kulathu, and the other, anonymous, reviewer for their contribution to the peer review of this work. Peer reviewer reports are available.

Reprints and permission information is available at http://www.nature.com/reprints

Publisher's note Springer Nature remains neutral with regard to jurisdictional claims in published maps and institutional affiliations.

\section{(i)}

Open Access This article is licensed under a Creative Commons Attribution 4.0 International License, which permits use, sharing, adaptation, distribution and reproduction in any medium or format, as long as you give appropriate credit to the original author(s) and the source, provide a link to the Creative Commons license, and indicate if changes were made. The images or other third party material in this article are included in the article's Creative Commons license, unless indicated otherwise in a credit line to the material. If material is not included in the article's Creative Commons license and your intended use is not permitted by statutory regulation or exceeds the permitted use, you will need to obtain permission directly from the copyright holder. To view a copy of this license, visit http://creativecommons.org/ licenses/by/4.0/

(C) The Author(s) 2019 